Probing dark matter decay and annihilation with Fermi LAT observations of nearby galaxy clusters

This article has been downloaded from IOPscience. Please scroll down to see the full text article.

JCAP01(2012)042

(http://iopscience.iop.org/1475-7516/2012/01/042)

View the table of contents for this issue, or go to the journal homepage for more

Download details:

IP Address: 131.169.95.134

The article was downloaded on 31/07/2012 at 14:30

Please note that terms and conditions apply. 


\title{
Probing dark matter decay and annihilation with Fermi LAT observations of nearby galaxy clusters
}

\author{
Xiaoyuan Huang, ${ }^{a, b}$ Gilles Vertongen ${ }^{c, d}$ and Christoph Weniger ${ }^{b}$ \\ ${ }^{a}$ National Astronomical Observatories, Chinese Academy of Sciences, \\ Beijing, 100012, China \\ ${ }^{b}$ Max-Planck-Institut für Physik, \\ Föhringer Ring 6, 80805 München, Germany \\ ${ }^{c}$ Deutsches Elektronen-Synchrotron (DESY), \\ Notkestrasse 85, 22603 Hamburg, Germany \\ ${ }^{d}$ Institut d'Astrophysique de Paris, UMR-7095 du CNRS, \\ 98 bis bd Arago, 75014 Paris, France \\ E-mail: huang@mppmu.mpg.de, gilles.vertongen@desy.de, \\ weniger@mppmu.mpg.de
}

Received October 18, 2011

Accepted December 23, 2011

Published January 23, 2012 
Abstract. Galaxy clusters are promising targets for indirect dark matter searches. Gammaray signatures from the decay or annihilation of dark matter particles inside these clusters could be observable with the Fermi Large Area Telescope (LAT). Based on three years of Fermi LAT gamma-ray data, we analyze the flux coming from eight nearby clusters individually as well as in a combined likelihood analysis. Concentrating mostly on signals from dark matter decay, we take into account uncertainties of the cluster masses as determined by $\mathrm{X}$-ray observations and model the cluster emission as extended sources. Searching for different hadronic and leptonic decay and annihilation spectra, we do not find significant emission from any of the considered clusters and present limits on the dark matter lifetime and annihilation cross-section. We compare our lifetime limits derived from cluster observations with the limits that can be obtained from the extragalactic gamma-ray background (EGBG), and find that in case of hadronic decay the cluster limits become competitive at dark matter masses below a few hundred GeV. In case of leptonic decay, however, galaxy cluster limits are stronger than the limits from the EGBG over the full considered mass range. Finally, we show that in presence of dark matter substructures down to $10^{-6}$ solar masses the limits on the dark matter annihilation cross-section could improve by a factor of a few hundred, possibly going down to the thermal cross-section of $3 \times 10^{-26} \mathrm{~cm}^{3} \mathrm{~s}^{-1}$ for dark matter masses $\lesssim 150 \mathrm{GeV}$ and annihilation into $b \bar{b}$. As a direct application of our results, we derive limits on the lifetime of gravitino dark matter in scenarios with $R$-parity violation. Implications of these limits for the possible observation of long-lived superparticles at the LHC are discussed.

Keywords: dark matter experiments, gamma ray experiments, dark matter theory

ArXiv ePrint: 1110.1529 


\section{Contents}

1 Introduction $\quad 1$

2 Galaxy cluster analysis $\quad 3$

2.1 Dark matter signal 4

2.2 Dark matter distribution 6

$\begin{array}{ll}2.3 \text { Signal boost from dark matter substructures } & 7\end{array}$

$\begin{array}{llr}2.4 \text { Data analysis } & 9\end{array}$

3 Limits from the EGBG $r$

4 Results $\quad 12$

5 Consequences for gravitino dark matter $\quad 18$

$\begin{array}{llr}5.1 & R \text {-parity breaking model } & 19\end{array}$

$\begin{array}{ll}5.2 & \text { Limits from galaxy clusters } \\ & 5.2 .1\end{array}$

5.2.1 $R$-parity breaking parameter 20

5.2.2 Stau NLSP decay length 22

5.2.3 Neutralino NLSP decay length 22

6 Conclusions 23

\section{Introduction}

Galaxy clusters are the most massive gravitationally collapsed objects in the Universe. Historically, the kinematical study of the Coma cluster provided the first indication for the existence of dark matter [1]. Further gravitational evidence for dark matter was found at many places, e.g. in stellar rotation curves of spiral galaxies or as baryon acoustic oscillations imprinted in the cosmic microwave background (for reviews on particle dark matter see refs. [2-4]). By now, the $\Lambda$ CDM scenario is the standard framework for cosmology, leaving open the question of what the nature of the dark matter particles is. Theoretical models for dark matter predict a large variety of possible non-gravitational signatures that would help to reveal its properties. However, despite lots of efforts, none of these signals has been unambiguously detected so far.

If dark matter is made out of WIMPs (Weakly Interacting Massive Particles), their efficient self-annihilation in the early Universe would explain the observed dark matter density. Today, the same annihilation process could contribute to the measured cosmic-ray fluxes; a clear detection of the annihilation products would reveal information about the dark matter particle's mass and interactions. Similar signatures could be produced if dark matter is unstable and decays, providing us with information on the lifetime of the dark matter particle. Indirect searches for dark matter are aiming at seeing such annihilation or decay signals above the astrophysical backgrounds. These searches typically concentrate on photons or neutrinos, which carry spatial and spectral information about their origin, and on different 
anti-matter species with their relatively low astrophysical backgrounds. Together with satellite galaxies of the Milky Way [5-11] and the Galactic center [12-19], nearby massive galaxy clusters are among the most promising targets for indirect dark matter searches by means of gamma rays [20-30].

A theoretically well motivated example for decaying dark matter is the gravitino $\psi_{3 / 2}$, which appears in locally supersymmetric extensions of the Standard Model. In scenarios where $R$-parity is mildly violated and the gravitino is the lightest superparticle (LSP), thermal leptogenesis, gravitino dark matter and primordial nucleosynthesis are naturally consistent [31]. Within this framework, the gravitino would decay with cosmological lifetimes [32], making its decay products potentially observable in the cosmic-ray fluxes [33-44]. For gravitino masses $\lesssim 100 \mathrm{GeV}$, the most prominent feature in the decay spectrum is an intense gamma-ray line, produced by the two-body decay into neutrinos and photons, $\psi_{3 / 2} \rightarrow \gamma \nu$ [34]. Dedicated searches for such a feature in the current gamma-ray observations of the Fermi LAT [45] exist in the literature, see refs. [46, 47], and their null results were used to put lower limits on the gravitino lifetime around $6 \times 10^{28} \mathrm{~s}$ [47]. However, for larger gravitino masses $\gtrsim 100 \mathrm{GeV}$, the branching ratio into gamma-ray lines is strongly suppressed, and instead decay modes like $\psi_{3 / 2} \rightarrow W^{ \pm} \ell^{\mp}$ and $\psi_{3 / 2} \rightarrow Z^{0} \nu$ produce a gamma-ray flux with a broad continuous energy spectrum. It is this flux that could potentially show up in observations of galaxy clusters, whereas the observation of the gamma-ray line in galaxy clusters would be difficult due to the limited statistics.

In general, dark matter lifetimes of the order of $10^{26}-10^{29} \mathrm{~s}$, which is in the ballpark of what is accessible experimentally, are obtained when the symmetry responsible for the dark matter stability is violated by dimension six operators generated close to the grand unification scale [48-50]. Indeed, models of this kind were proposed to explain the $e^{ \pm}$"excesses" observed by the PAMELA [51], Fermi LAT $[52,53]$ and H.E.S.S. $[54,55]$ experiments. To avoid the stringent anti-proton limits $[51,56]$, the decay should be mostly leptophilic (see e.g. refs. [57$63]$ ), and one typical decay mode that could well reproduce the locally observed $e^{ \pm}$fluxes is the decay into muons, $\psi \rightarrow \mu^{+} \mu^{-}$, with a large dark matter mass around $m_{\psi} \simeq 3 \mathrm{TeV}$ and lifetimes around $2 \times 10^{26} \mathrm{~s}[64,65]$. Inside galaxy clusters, due to inverse Compton scattering (ICS) on the cosmic microwave background (CMB), almost all of the kinetic energy of the produced high-energetic electrons and positrons is transferred into gamma rays, with energies up to $\mathcal{O}(100 \mathrm{GeV})$. This makes possible the investigation of the decaying and annihilating dark matter interpretations of the $e^{ \pm}$excesses by galaxy cluster observations.

A dedicated search for dark matter annihilation signals from galaxy clusters, using 11 months of Fermi LAT data, was carried out in ref. [26]. The null result of this search was used to derive limits on the dark matter annihilation rate into $b \bar{b}$ and $\mu^{-} \mu^{+}$. In ref. [27] these results were translated into limits on the dark matter decay rate, and it was demonstrated that galaxy cluster observations give strong constraints on the dark matter lifetime, superior to the limits that could be obtained from satellite galaxy observations, and of the order of the limits that can be derived from the extragalactic gamma-ray background. This makes galaxy clusters promising targets when searching for signals from dark matter decay. Concerning WIMP dark matter, taking into account the expected boost of the annihilation signal due to dark matter substructures in the cluster halo, limits can potentially go down to the crosssection expected from thermal freeze-out [29, 30]. Further studies of the galaxy cluster emission as seen by the Fermi LAT, H.E.S.S. and MAGIC were presented in ref. [23, 24, 28], some applications to annihilating and decaying dark matter models were discussed in refs. [66-68]. 
Besides the large amount of dark matter, it is known from radio observations that galaxy clusters are also a host for energetic cosmic rays, which can be accelerated during the process of cluster formation by mergers or accretion shocks. Proton-proton collision as well as the ICS of an energetic electron population can produce a possibly observable gamma-ray flux (see e.g. refs. $[26,30]$ ). Such a flux should however be finally distinguishable from a dark matter signal through the analysis of the energy spectra if the statistics is high enough [21].

In this paper, we analyze the gamma-ray flux from eight galaxy clusters as measured by the Fermi LAT since Aug 2008, and we present constraints on the dark matter lifetime and annihilation cross-section. We analyze the different target clusters individually as well as in a combined likelihood approach, and search for significant gamma-ray emission as an indication for decaying or annihilating dark matter. In ref. $[26,27]$ the dark matter signal was approximated to be point-source like. Importantly, this approximation becomes problematic in the case of dark matter decay or substructure-boosted annihilation, since the extend of the expected signal starts to exceed the angular resolution of the Fermi LAT. To account for this, we model the dark matter emission as an extended source. In absence of a clear signal, we derive limits on the dark matter lifetime and annihilation cross-section as function of the dark matter mass, for different hadronic and leptonic final states. Cluster masses and the expected decay or annihilation signals are derived from the extended HIFLUGCS catalog $[69,70]$ which is based on ROSAT PSPC X-ray observations [71], and the corresponding uncertainties are consistently taken into account. We compare the obtained lifetime limits with the limits that can be derived from the extragalactic gamma-ray background, and we discuss the implications of our limits on the decaying or annihilating dark matter interpretation of the $e^{ \pm}$excesses. Furthermore, we will illustrate how the limits improve when a boost of the annihilating signal due to substructures is included. Finally, we apply our findings to the scenario of decaying gravitino dark matter, and derive new constraints on the gravitino lifetime for masses above about $100 \mathrm{GeV}$. We comment on implications for the possible observation of long-lived superparticles at the LHC.

The rest of the paper is organized as follows: in section 2 we present our galaxy cluster analysis of the Fermi LAT data. We discuss the expected dark matter signals, our treatment of the LAT data and the details of our statistical analysis. In section 3, we shortly review how limits on the dark matter lifetime from the extragalactic gamma-ray background are obtained. Our results and their discussion are presented in section 4. Finally, section 5 is devoted to gravitino dark matter, where we present limits on the gravitino lifetime for gravitino masses above $100 \mathrm{GeV}$, as well as the implied limits on the decay lengths of nextto-lightest superparticles (NLSP) at particle colliders. We conclude in section 6 .

Throughout this work we assume a $\Lambda \mathrm{CDM}$ cosmology with the parameters $\Omega_{\Lambda}=0.728$ and $h \equiv H_{0} / 100 \mathrm{~km} \mathrm{~s}^{-1} \mathrm{Mpc}^{-1}=0.704$ [72].

\section{Galaxy cluster analysis}

The eight galaxy clusters that we consider in this work are summarized in table 1 . They are selected from the extended HIFLUGCS X-ray catalog $[69,70]$ in order to yield large signals from dark matter decay, but are also among the best clusters when searching for signals from dark matter annihilation. Galaxy clusters with potentially large signals that we disregard are: Ophiuchus, A3627 and 3C129 because they lie too close to the Galactic plane, Centaurus, M49 and A2877 because of issues with our adopted model for Galactic diffuse 


\begin{tabular}{|lcccccc|}
\hline Cluster & R.A. & Dec. & $\mathrm{z}$ & $\begin{array}{c}J_{\Delta \Omega}^{\text {dec. }} \\
{\left[10^{18} \mathrm{GeV} \mathrm{cm}^{-2}\right]}\end{array}$ & $\begin{array}{c}J_{\Delta \Omega}^{\text {ann. }} \\
{\left[10^{17} \mathrm{GeV}^{2} \mathrm{~cm}^{-5}\right]}\end{array}$ & $\begin{array}{c}\theta_{s} \\
{\left[{ }^{\circ}\right]}\end{array}$ \\
\hline Fornax & 54.67 & -35.31 & 0.0046 & $20.3_{-6.8}^{+4.6}$ & $8.8_{-2.8}^{+2.0}$ & $0.44_{-0.11}^{+0.07}$ \\
Coma & 194.95 & 27.94 & 0.0232 & $10.7_{-2.7}^{+1.8}$ & $1.3_{-0.31}^{+0.20}$ & $0.23_{-0.04}^{+0.02}$ \\
A1367 & 176.19 & 19.70 & 0.0216 & $10.6_{-2.9}^{+1.3}$ & $1.4_{-0.34}^{+0.35}$ & $0.23_{-0.04}^{+0.02}$ \\
A1060 & 159.18 & -27.52 & 0.0114 & $10.2_{-3.5}^{+2.0}$ & $2.2_{-0.69}^{+0.38}$ & $0.24_{-0.06}^{+0.03}$ \\
AWM7 & 43.62 & 41.58 & 0.0172 & $9.9_{-3.9}^{+1.9}$ & $1.6_{-0.56}^{+0.27}$ & $0.22_{-0.06}^{+0.03}$ \\
S636 & 157.52 & -35.31 & 0.0116 & $6.8_{-1.7}^{+1.5}$ & $1.5_{-0.34}^{+0.29}$ & $0.18_{-0.03}^{+0.03}$ \\
NGC4636 & 190.71 & 2.69 & 0.0037 & $6.1_{-1.7}^{+0.80}$ & $3.5_{-0.85}^{+0.39}$ & $0.19_{-0.04}^{+0.02}$ \\
NGC5813 & 225.30 & 1.70 & 0.0064 & $6.0_{-4.2}^{+4.6}$ & $2.2_{-1.4}^{+1.4}$ & $0.18_{-0.10}^{+0.08}$ \\
\hline
\end{tabular}

Table 1. Galaxy clusters considered in this work, with their coordinates (equatorial J2000.0) and redshift $z$ from ref. [69]. We show the integrated surface densities $J_{\Delta \Omega}^{\text {dec. }} \equiv \int_{\Delta \Omega} J^{\text {dec. }}(\Omega)$ and $J_{\Delta \Omega}^{\text {ann. }} \equiv$ $\int_{\Delta \Omega} J^{\text {ann. }}(\Omega)$ of the dark matter signal, obtained inside a region of $1^{\circ}$ radius around the cluster center, as well as the projected angle $\theta_{s}$ of the scale radius $r_{s}$ of the adopted NFW profile. Central values and errors for these parameters are derived from the cluster masses in ref. [70].

emission, ${ }^{1}$ and Virgo (M87) and Perseus because of the presence of bright gamma-ray sources at their center [73, 74].

\subsection{Dark matter signal}

The gamma-ray flux from dark matter annihilation or decay that is expected to be seen in galaxy cluster observations factorizes into an astrophysical part, which contains information about the dark matter distribution $\rho_{\mathrm{dm}}$, and a particle-physics part, which is universal for all observed targets. Assuming a spherical dark matter halo, the astrophysical factor, $J^{\text {dec./ann. }}(\theta)$, just depends on the cluster-centric angle $\theta$ and is given by a line-of-sight integral. In the case of dark matter decay, the signal flux reads

$$
\frac{d J_{\mathrm{sig}}}{d E d \Omega}(\theta)=\frac{1}{4 \pi m_{\psi} \tau_{\psi}} \frac{d N_{\gamma}}{d E} \underbrace{\int_{\text {l.o.s. }} d s \rho_{\mathrm{dm}}(s, \Omega)}_{\equiv J^{\text {dec. }}(\theta)},
$$

while in the annihilation case, it is given by

$$
\frac{d J_{\mathrm{sig}}}{d E d \Omega}(\theta)=\frac{\langle\sigma v\rangle}{8 \pi m_{\psi}^{2}} \frac{d N_{\gamma}}{d E} \underbrace{\int_{\text {l.o.s. }} d s \rho_{\mathrm{dm}}^{2}(s, \Omega)}_{\equiv J^{\text {ann. }}(\theta)} .
$$

Here, $m_{\psi}$ denotes the dark matter mass, while $\tau_{\psi}$ and $\langle\sigma v\rangle$ are the dark matter lifetime and total annihilation cross section, respectively. The energy spectrum of gamma rays produced in the decay/annihilation is given by $d N_{\gamma} / d E$. Note that the energy spectrum $d N_{\gamma} / d E=$ $d N_{\gamma}^{\text {prim }} / d E+d N_{\gamma}^{\mathrm{IC}} / d E$ includes prompt gamma rays that are directly produced in the decay

\footnotetext{
${ }^{1}$ The positions of the Centaurus and M49 clusters unfortunately coincide with sharp edges in our Galactic diffuse emission model, gal_2yearp7v6_v0; the region near A2877 contains a large number of faint sources that are not part of the 2FGL. In all three cases the background fits are unreliable, and we neglect these targets from our analysis. Including them would improve our overall limits.
} 
or annihilation process (final-state radiation, $\pi^{0} \rightarrow \gamma \gamma$ etc.) as well as the gamma rays that originate from ICS losses of $e^{ \pm}$from dark matter on the intra-cluster radiation field. We calculated the energy spectra of gamma rays and electrons with the event generator Pythia 6.4.19 [75], and cross-checked our results with the analytic expressions presented in ref. [76].

Inverse Compton scattering. Electrons and positrons produced in the decay or annihilation of dark matter particles inside galaxy clusters suffer inverse Compton and synchrotron losses when interacting with the intra-cluster radiation field. The dominant component of this radiation field is in most cases the CMB; other contributions, which can become relevant close to the cluster center, are the starlight, dust radiation and the intra-cluster magnetic field (see discussion in ref. [30]). In case of dark matter decay or substructure-boosted annihilation, the possible impact of these additional components on our results is small, as we will exemplify below for the Coma cluster; if not stated otherwise, we consider the CMB only throughout this work.

The average energy spectrum of gamma-rays with energy $E_{\gamma}$ that are generated by the inverse Compton scattering of one electron with an initial energy of $E_{0}$ is given by

$$
\frac{d N_{\gamma}^{\mathrm{IC}}}{d E_{\gamma}}=\int_{0}^{\infty} d \epsilon \int_{m_{e}}^{E_{0}} d E_{e} \frac{d \sigma^{\mathrm{IC}}\left(E_{e}, \epsilon\right)}{d E_{\gamma}} \frac{f_{\mathrm{CMB}}(\epsilon)}{b_{\mathrm{loss}}\left(E_{e}\right)} .
$$

Here, $f_{\mathrm{CMB}}(\epsilon)$ is the $\mathrm{CMB}$ energy spectrum with temperature $T_{\mathrm{CMB}}=2.725 \mathrm{~K}$, and $d \sigma^{\mathrm{IC}} / d E_{\gamma}$ denotes the differential cross section of inverse Compton scattering of an electron with energy $E_{e}$ when a CMB photon with energy $\epsilon$ is up-scattered to energies between $E_{\gamma}$ and $E_{\gamma}+d E_{\gamma}$. Due to the very low energy of the CMB photons, the center-of-mass energy of the processes we are interested in is always smaller than the electron mass, which allows us to use the non-relativistic limit of the Klein-Nishina equation in our calculations ${ }^{2}$ (see e.g. ref. [77]):

$$
\frac{d \sigma^{\mathrm{IC}}\left(E_{e}, \epsilon\right)}{d E_{\gamma}}=\frac{3}{4} \frac{\sigma_{\mathrm{T}}}{\gamma_{e}^{2} \epsilon}\left[2 q \ln q+1+q-2 q^{2}\right]
$$

where $\sigma_{\mathrm{T}}=0.67$ barn is the Compton scattering cross section in the Thomson limit, $\gamma_{e} \equiv$ $E_{e} / m_{e}$ is the Lorentz factor of the electron, $m_{e}=511 \mathrm{keV}$ is the electron mass, and $q \equiv$ $E_{\gamma} / E_{\gamma}^{\max }$ with $E_{\gamma}^{\max } \equiv 4 \gamma_{e}^{2} \epsilon$. Eq. (2.4) holds in the range $\epsilon \leq E_{\gamma} \leq E_{\gamma}^{\max }$, in the limit where down-scattering is neglected.

In the above equation, $b_{\text {loss }}\left(E_{\mathrm{e}}\right)$ is the energy loss rate of an electron with energy $E_{\mathrm{e}}$. Typically, the main contribution comes from ICS on the CMB, but we can also include synchrotron losses on the intra-cluster magnetic field; $b_{\mathrm{loss}}=b_{\mathrm{ICS}}+b_{\mathrm{syn}}$. In the non-relativistic limit, the ICS and synchrotron losses read

$$
b_{\mathrm{ICS}}\left(E_{e}\right)=\frac{4}{3} \sigma_{\mathrm{T}} \gamma_{e}^{2} \underbrace{\int_{0}^{\infty} d \epsilon \epsilon f_{\mathrm{CMB}}(\epsilon)}_{\equiv \rho_{\mathrm{CMB}}} \text { and } b_{\mathrm{syn}}\left(E_{e}\right)=\frac{4}{3} \sigma_{\mathrm{T}} \gamma_{e}^{2} \frac{B^{2}}{2},
$$

respectively. In order for the magnetic field $B$ to dominate the $\mathrm{CMB}$ energy density $\rho_{\mathrm{CMB}}$ (namely, $B^{2} / 2>\rho_{\mathrm{CMB}}$ ) in galaxy clusters it has to exceed the critical value $B_{\mathrm{CMB}}=3.2 \mu \mathrm{G}$ (assuming redshifts $z \ll 1$ ). The energy spectrum of the dark matter induced ICS radiation would then scale like $\propto\left(1+(B / 3.2 \mu \mathrm{B})^{2}\right)^{-1}$.

\footnotetext{
${ }^{2}$ This approximation breaks down for electron energies above $m_{e}^{2} / T_{\mathrm{CMB}} \sim 10^{3} \mathrm{TeV}$.
} 
The energy loss time $\tau_{\text {loss }}=E_{e} / \dot{E}_{e}$ of electrons with $100 \mathrm{GeV}-10 \mathrm{TeV}$ energies, as relevant for our work, lies in the range of $10^{-4}-10^{-2} \mathrm{~Gy}$. This is much shorter than the cosmic-ray relaxation times in galaxy clusters which typically are of order 1-10 Gy [30, 78]. The propagation scale corresponding to $\gtrsim 100 \mathrm{GeV}$ electrons is expected to be $\lesssim 1 \mathrm{kpc}[79,80]$, which for Mpc distances is well below the angular resolution of the LAT [81]. We hence neglect effects of cosmic-ray transport and consider that $e^{ \pm}$are loosing all their energy instantaneously where they are produced. In this limit, the angular profile of the ICS signal is identical to the angular profile of the prompt radiation. This is opposite to the case of dwarf galaxies, where due to their proximity propagation effects have to be taken into account in general, see e.g. ref. [8].

After some algebra, one can finally show that the energy spectrum of ICS radiation emitted from a single electron with an initial energy $E_{e}$ is given by the expression $(B \rightarrow 0)$

$$
\frac{d N_{\gamma}^{\mathrm{IC}}}{d E_{\gamma}}=\frac{9 m_{e}}{32 \gamma_{e}^{3}} \int_{\frac{1}{4 \gamma_{e}^{2}}}^{1} d q \frac{1}{q^{5 / 2}}\left\{\frac{92}{525}-\frac{2}{3} q^{3 / 2}-\frac{2}{25} q^{5 / 2}+\frac{4}{7} q^{7 / 2}-\frac{4}{5} q^{5 / 2} \log q\right\} \frac{f_{\mathrm{CMB}}\left(\frac{E_{\gamma}}{4 \gamma_{e}^{2} q}\right)}{\rho_{\mathrm{CMB}}}
$$

A subsequent convolution with the energy spectrum $d N_{e} / d E_{e}$ of electrons and positrons yields then the ICS contribution to the gamma-ray spectrum, $d N_{\gamma}^{\mathrm{IC}} / d E$, in eqs. (2.1) and (2.2).

\subsection{Dark matter distribution}

As discussed above, the expected dark matter signals depend crucially on the dark matter profiles $\rho(r)_{\mathrm{dm}}$ of the target galaxy clusters. We assume throughout this work that the smooth component of the dark matter halo follows a Navarro-Frenk-White (NFW) profile [46, 82],

$$
\rho_{\mathrm{dm}}(r)=\frac{\rho_{s}}{r / r_{s}\left(1+r / r_{s}\right)^{2}},
$$

where the scale radius $r_{s}$ and the density normalization $\rho_{s}$ have to be determined from observations. ${ }^{3}$ The cluster mass $M_{\Delta}$ inside a cluster-centric radius $r_{\Delta}$ is defined such that the average density inside $r_{\Delta}$ equals $\Delta$ times the critical density of the Universe, $\rho_{c}$ (typically $\Delta \approx 100-500$ ). To determine the parameters of the NFW profile from $M_{\Delta}$ and $r_{\Delta}$, we adopt the observationally obtained concentration-mass relation from ref. [85],

$$
c_{\mathrm{vir}}\left(M_{\mathrm{vir}}\right)=9\left(\frac{M_{\mathrm{vir}}}{10^{14} h^{-1} M_{\odot}}\right)^{-0.172},
$$

where the concentration parameter $c \equiv r_{\mathrm{vir}} / r_{s}$ relates the virial radius $r_{\mathrm{vir}}$ as defined by $\Delta=\Delta_{\text {vir }} \simeq 98$ (see appendix of ref. [86] and references therein) to the scaling radius $r_{s}$, and $M_{\odot}=2.0 \times 10^{30} \mathrm{~kg}$ denotes the solar mass. ${ }^{4}$ The virial radius $r_{\mathrm{vir}}$ is then related to $r_{\Delta}$ via $[86]$

$$
f\left(r_{s} / r_{\Delta}\right)=\frac{\Delta}{\Delta_{\text {vir }}} f\left(r_{s} / r_{\text {vir }}\right)
$$

\footnotetext{
${ }^{3}$ We find that using an Einasto profile $[83,84]$ with similar $M_{200}$ and $r_{200}$ (with $\alpha=0.17, r_{-2}=r_{s}$ and $\rho_{-2} \simeq \rho_{s} / 4.2$ [30]) leaves the results for decaying dark matter essentially unchanged, whereas the fluxes from dark matter annihilation as summarized in table 1 are increased by about $\sim 30 \%$.

${ }^{4}$ Varying the prefactor in $c_{\mathrm{vir}}$ in the range $9 \pm 2$ leaves the fluxes from decaying dark matter essentially unchanged, whereas the annihilation fluxes increase or decrease by about 30-40\%. Note that CDM simulations favor a somewhat smaller concentration of about $\sim 6$ at $M_{\mathrm{vir}}=10^{14} h^{-1} M_{\odot}[87,88]$.
} 

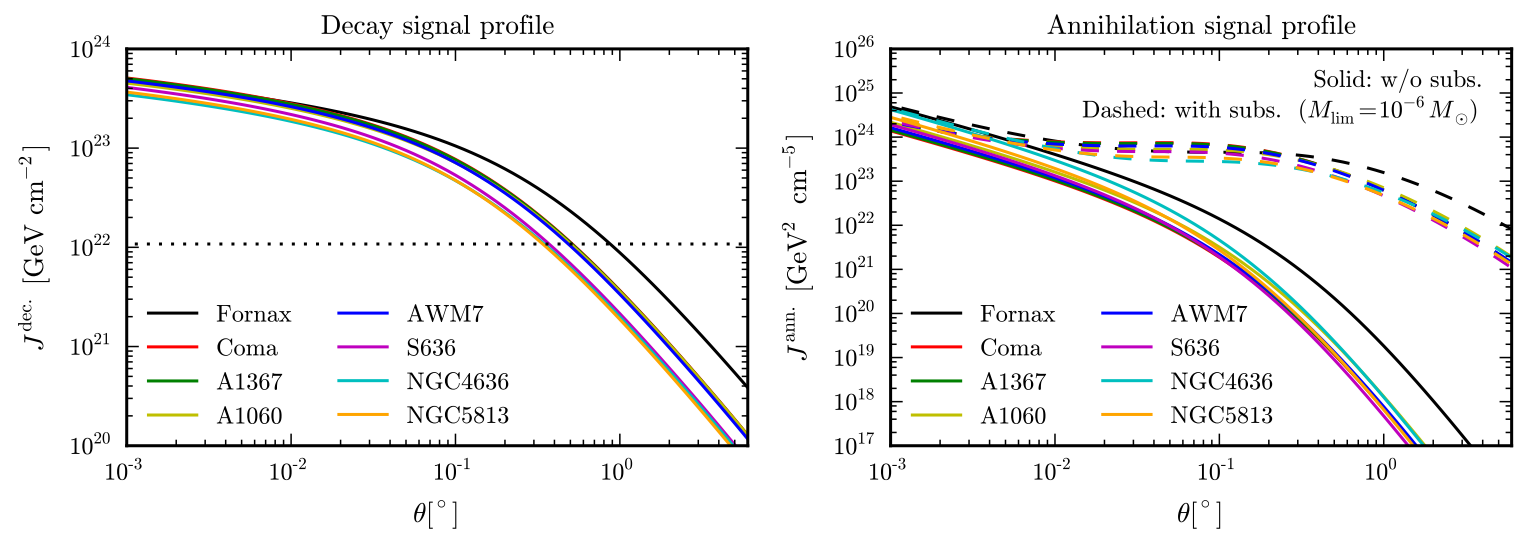

Figure 1. Left panel: profile of the dark matter decay signal as function of the cluster-centric angle $\theta$ (PSF effects not included). The dotted line shows the isotropic Galactic contribution to the dark matter signal. Right panel: the same for the dark matter annihilation signal. Solid lines show the signal coming from the smooth halo component alone, dashed lines include effects from dark matter substructures, which boosts the signal at angles around $1^{\circ}$ (as discussed in section 2.3). For comparison: the angular resolution of the LAT (P7SOURCE_V6) in terms of the $68 \%$ containment angle is $6^{\circ}$ at $100 \mathrm{MeV}, 0.9^{\circ}$ at $1 \mathrm{GeV}$ and $0.2^{\circ}$ at $100 \mathrm{GeV}[81]$.

where

$$
f(x)=x^{3}\left[\ln \left(1+x^{-1}\right)-(1+x)^{-1}\right]
$$

and

$$
\frac{M_{\Delta}}{M_{\text {vir }}}=\frac{\Delta}{\Delta_{\text {vir }}}\left(\frac{r_{\Delta}}{r_{\text {vir }}}\right)^{3}
$$

With eqs. (2.8), (2.9) and (2.11), one can find $r_{s}$ and $\rho_{s}$ as a function of $M_{\Delta}$ and $r_{\Delta}$.

Using the cluster masses as derived from ROSAT PSPC X-ray observations in the extended HIFLUGCS catalog [70], we calculate the signal surface densities $J^{\text {ann. }}$ and $J^{\text {dec. }}$ as described above. ${ }^{5}$ Our results, as function of the cluster-centric angle $\theta$, are plotted in figure 1. These profiles are used to model the extended dark matter signal in our analysis. For convenience and comparison with previous work, we show results for $J^{\text {ann. }}$ and $J^{\text {dec. }}$ integrated over a cluster-centric region of $1^{\circ}$ radius in table 1 . There, we also indicate the projected scaling angle $\theta_{s}=r_{s} / D$, where the distance to the cluster is given by $D \simeq z c / H_{0}$. The signal uncertainties shown in table 1 are directly derived from the mass uncertainties in ref. [70] and as large as a factor of two in some cases. Within the error bars our results agree largely with what was found in refs. [26, 27] based on the initial HIFLUGCS catalog [69]. Besides the uncertainties from the fits to the X-ray profiles that were already discussed in ref. [69], the mass ranges quoted in ref. [70] take additionally into account uncertainties in the $\mathrm{X}$-ray temperature profile, which leads to somewhat larger error-bars compared to ref. [69].

\subsection{Signal boost from dark matter substructures}

A prediction of the cold dark matter paradigm is the hierarchical structuring of dark matter halos. Dark matter substructures inside of galaxy cluster halos are observationally known to

\footnotetext{
${ }^{5}$ The values of $M_{500}$ and $r_{500}$ are rescaled to our adopted Hubble constant, and $M_{500}$ is reduced by the gas fraction $f_{\text {gas }}$ indicated in ref. [70]. We checked that using $M_{200} \simeq \sqrt{5 / 2} M_{500}$ and $r_{200} \simeq \sqrt{5 / 2} r_{500}$ as a starting point would increase the predicted fluxes by $10-20 \%$.
} 
exist down to the scale of dwarf galaxies, $10^{7} M_{\odot}$; for thermally produced WIMPs they are predicted to continue down to free streaming masses of about $10^{-6} M_{\odot}$ and below $[89,90]$ (for a discussion of possible ranges depending on the dark matter model see ref. [91]). Since the dark matter annihilation signal depends on the dark matter density squared, the existence of substructures can boost the annihilation signal considerably with respect to the signal from the smooth halo; the details depend on the mass function of substructures, the concentration mass relation and the radial distribution (see e.g. ref. [30] for a recent discussion). Dynamical friction and tidal stripping near the cluster center lead to a local depletion of substructures that results in a relative enhancement of the boosted signal in the outskirts of the main halo. In general, the boosted signal is expected to be considerably more extended than the signal coming from the smooth main halo alone. Deriving the magnitude of the signal boost relies on extrapolations of numerical simulations for dissipationless DM [92-94] over many orders of magnitude in the substructure mass. In the literature, predictions for the substructure boost have not yet converged; in case of galaxy clusters, signal boosts in the range of $\sim 10-50[26,29]$ up to $\sim 1000[25,30]$ were recently discussed. As mentioned above, the actual values strongly depend on the adopted subhalo mass fraction (which is partially correlated with the value of $\sigma_{8}$ used in the underlying simulations), the subhalo mass distribution functions and the adopted halo concentration. Furthermore, for individual clusters, precise predictions appear to be difficult since the halo-to-halo scattering of the substructure fraction, which roughly correlates with the concentration of the particular halo, can be quite large and $\mathcal{O}(1)$ [95].

In the present paper, we adopt an optimistic scenario and estimate the boosted dark matter signal following ref. [30]: based on the high-resolution dissipationless dark matter simulations of the Aquarius project [92] (which features a realtively large subhalo fraction), the boost of the dark matter annihilation signal was determined in ref. [93] for a Milky Way sized halo. Extrapolating the mass of the smallest subhalos down to $M_{\text {lim }}=10^{-6} M_{\odot}$, an increase in the overall luminosity $L=\int d V \rho_{\mathrm{dm}}^{2}$ of about 230 was found, being mostly due to a signal enhancement at large galactocentric distances. The luminosity due to substructures inside a radius $r$ is well fitted by [30]

$$
\begin{aligned}
L_{\mathrm{sub}}(<r) & =a_{0} C\left(M_{200}\right) L_{200 \mathrm{sm}}\left(M_{200}\right) x^{f(x)}, \\
f(x) & =a_{1} x^{a_{2}}, \\
C\left(M_{200}\right) & =0.023\left(\frac{M_{200}}{M_{\mathrm{lim}}}\right)^{\alpha_{C}},
\end{aligned}
$$

where $x \equiv r / r_{200}, \alpha_{0}=0.76, \alpha_{1}=0.95, \alpha_{2}=-0.27$ and $\alpha_{C}=0.226$. Here, $L_{200 \mathrm{sm}}$ denotes the luminosity of the smooth halo component inside $r_{200}$ alone. The only free parameter is the cutoff scale for the dark matter subhalo mass, which we fix to $M_{\lim }=10^{-6} M_{\odot}$. The parameters $M_{200}$ and $r_{200}$ are directly determined from the adopted NFW profile for each cluster. The overall boosted dark matter signal can then be calculated from eq. (2.12).

Our results for the signal profile from dark matter annihilation in presence of substructures are shown in the right panel of figure 1 by dashed lines, the signal from the smooth halo alone is shown by solid lines. In presence of substructures, the annihilation signal extends to radii of around $1^{\circ}$, below $0.01^{\circ}$ it is still dominated by the smooth dark matter halo. The boost factors that we obtain for the different considered galaxy clusters inside an opening angle of $\theta_{200}=r_{200} / D$ are in the range 500-1200, in agreement with ref. [30]. Similar large values were recently also found in ref. [96] (however, see discussion above). For different values of the cutoff $M_{\text {lim }}$ the boosted signal scales like $\propto M_{\text {lim }}^{-0.226}$, whereas its angular profile remains unchanged. 


\subsection{Data analysis}

The gamma-ray events entering our analysis are selected from the P7SOURCE_V6 event class of the Fermi LAT data measured between 4 Aug 2008 and 21 Jul 2011. ${ }^{6}$ From all events recorded by the Fermi LAT, we select those with energies between $400 \mathrm{MeV}$ and $100 \mathrm{GeV}$ and apply the zenith angle criterion $\theta<100^{\circ}$ in order to avoid contamination by the Earth's Albedo. ${ }^{7}$ For each galaxy cluster, we consider photons events in a $10^{\circ} \times 10^{\circ}$ squared region centered on the cluster position. These events are binned into a cube of $0.1^{\circ} \times 0.1^{\circ}$ pixels with 24 logarithmic energy bins. The lower end of the considered energy range is somewhat larger than what was used in previous works, e.g. refs. [8, 26]: below energies of $400 \mathrm{MeV}$, the point spread function (PSF) of the LAT becomes of the size of our considered target regions [81], whereas in the considered dark matter scenarios no relevant gamma-ray fluxes below $\sim 400 \mathrm{MeV}$ are expected; this motivates our choice.

For the diffuse background fluxes we take the isotropic emission and the galactic foreground model templates currently advocated by the Fermi LAT collaboration for point source analysis (iso_p7v6source and gal_2yearp7v6_v0). The galactic foreground model contains several dedicated spatial templates to model diffuse emission that is not accounted for by the GALPROP code (e.g. for Loop I and the Galactic Lobes [97]). ${ }^{8}$ These spatial templates exhibit sharp edges, and we exclude clusters that coincide with these edges (Centaurus and M49) from our analysis in order to avoid a bias of our results. On top of the diffuse templates, we add the point sources from the second Fermi LAT catalog 2FGL [98]. We include all point sources within a radius of $12^{\circ}$ around the cluster centers. Some of the sources lie outside of our target regions but might still contribute due to the large point-spread function (PSF) of the Fermi LAT at low energies. We furthermore (and conservatively for the purpose of deriving limits) assume that gamma-ray emission due to e.g. shock accelerated cosmic rays inside the cluster is absent and attribute possible observed fluxes entirely to dark matter.

We use the profile likelihood method to fit the data and derive limits $[99,100]$. The corresponding likelihood function $\mathcal{L}$ is - for an individual clusters $j$ - given by $\mathcal{L}_{j}\left(\boldsymbol{c}^{j} \mid \boldsymbol{\mu}^{j}\right)=$ $\Pi_{i} P\left(c_{i}^{j} \mid \mu_{i}^{j}\right)$, where $P(c \mid \mu)$ denotes the Poisson probability to observe $c$ events when $\mu$ are expected. The number of expected counts $\mu_{i}^{j}=\mu_{i}^{j}(\boldsymbol{\alpha})$ that is predicted for an energy/spatial bin $i$ is a function of the model parameters $\boldsymbol{\alpha}$. These numbers are in principle obtained by a convolution of the above model fluxes with the instrument response function of the Fermi LAT. In this work, the convolution with the PSF is done using gtsrcmaps from the Fermi Science Tools. Like in most of the existing analyses of Fermi LAT data (for exceptions see refs. $[6,46,47])$ we will neglect the small but finite energy dispersion of the LAT, which would not significantly affect the broad energy spectra that we are considering. Finally, the best-fit model parameters for cluster $j$ are obtained by maximizing $\mathcal{L}_{j}\left(\boldsymbol{c}^{j} \mid \boldsymbol{\mu}^{j}(\boldsymbol{\alpha})\right)$ with respect to the model parameters $\boldsymbol{\alpha}$.

As a first step, we fit the data extracted from our eight target regions with the background model only. The free parameters in the fit are the normalizations of the two diffuse background templates, as well as the normalization and spectral index of all point sources inside a $5^{\circ}$ radius around the cluster position or with $T S$-values larger than 9 . In figure 2

\footnotetext{
${ }^{6}$ The event data as well as the corresponding information about the instrument response functions P7V6 can be obtained from http://fermi.gsfc.nasa.gov/ssc/data/. We checked that using the event class P7CLEAN_6 instead leads to results that are similar to what is presented in this paper.

${ }^{7}$ These selections are made using the Fermi Science Tools v9r23p1. For the cuts in gtmktime we took DATA_QUAL==1 as well as the RIO-based zenith angle cut.

${ }^{8}$ See http://fermi.gsfc.nasa.gov/ssc/data/access/lat/Model_details/Pass7_galactic.html.
} 

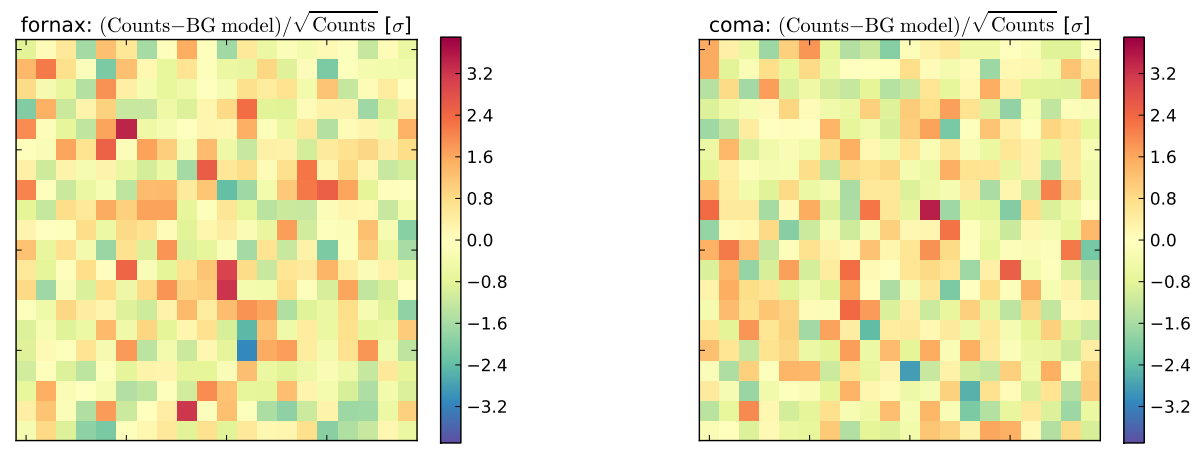

Figure 2. Residual maps after subtraction of our best-fit background models, in units of $1 \sigma$ standard deviations, for the case of the Fornax and Coma cluster. The maps span a $10^{\circ} \times 10^{\circ}$ region and are centered on the cluster position, pixels are resampled to $0.5^{\circ} \times 0.5^{\circ}$, counts are summed over the full energy range $400 \mathrm{MeV}-100 \mathrm{GeV}$. The count number per resampled pixel ranges between 11 and 273 with an average of 28.0 .
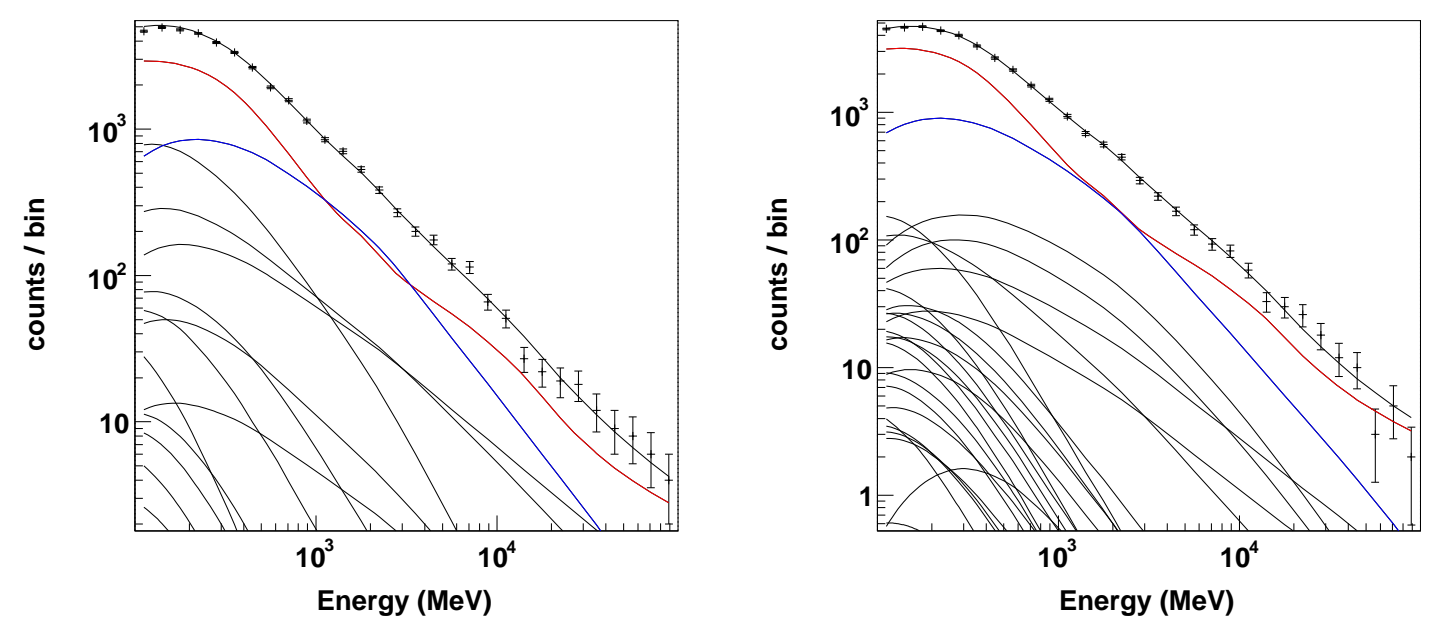

Figure 3. Energy spectra of different background components compared with data in the energy range $100 \mathrm{MeV}-100 \mathrm{GeV}$, for Fornax (left panel) and Coma cluster (right panel). Blue and red lines correspond to our two diffuse templates gal_2yearp7v6_v02 and iso_p7v6source, respectively, the lower black lines show the contribution from different point sources of the 2FGL. When performing the fits, we only use data down to $400 \mathrm{MeV}$, but the background models continuously connect also to data at lower energies, as shown in the plot.

we show for two exemplary clusters the residual maps that we obtain after subtracting our best-fit background models from the data, integrated over all energies; the corresponding energy spectra of the individual background components are shown in figure 3. For other clusters, we obtain similar results. The figures indicate that the adopted background models are sufficient to model the observations.

We then include the potential dark matter signals in the fits. The individual cluster signals are modeled as extended sources; their surface densities follow from eqs. (2.1) and (2.2) and are plotted in figure 1. We neglect the smooth Galactic and extragalactic components (cp. figure 1, dotted line) of the dark matter signal and assume that they are already accounted 
for by our two diffuse templates. ${ }^{9}$ Uncertainties of the cluster masses as given in ref. [70] translate into uncertainties on the integrated signal from each cluster, and into uncertainties on its angular shape as parametrized by $\theta_{s}$; the resulting errors are indicated in table 1 . We find that variations in $\theta_{s}$ have much less impact on our limits than variations of the integrated signal (less than $10 \%$ for the ranges given in table 1 ); for simplicity we will keep $\theta_{s}$ at its central value when performing fits to the data.

We include uncertainties of the cluster mass as a systematic error into the profile likelihood method by substituting the likelihood function of an individual cluster $\mathcal{L}_{j}$ (with a dark matter signal modeled according to the central values of table 1) with a likelihood function that takes into account the corresponding uncertainties of the integrated dark matter signal $[11]$

$$
\mathcal{L}_{j}\left(\alpha_{\mathrm{DM}}\right) \rightarrow \overline{\mathcal{L}}_{j}\left(\alpha_{\mathrm{DM}}\right) \equiv \max _{J_{\Delta \Omega}^{j}} \mathcal{L}_{j}\left(\alpha_{\mathrm{DM}} \frac{J_{\Delta \Omega}^{j}}{\bar{J}_{\Delta \Omega}^{j}}\right) \mathcal{L}_{j}^{\Delta M}\left(J_{\Delta \Omega}^{j}\right) .
$$

Here, $\alpha_{\mathrm{DM}}$ denotes the normalization of the dark matter signal (being related to the dark matter lifetime or annihilation cross-section), $J_{\Delta \Omega}^{j}$ is the integrated surface density, $\bar{J}_{\Delta \Omega}^{j}$ its central value as given in table 1 , and $\mathcal{L}_{j}^{\Delta M}$ is the likelihood function of $J_{\Delta \Omega}^{j}$ for cluster $j$. In this work, we approximate $\mathcal{L}_{j}^{\Delta M}$ by a log-normal distribution that is defined according to the error bars in table 1 in order to model the uncertainties of $J_{\Delta \Omega}^{j}$. To this end, we fix $\mathcal{L}_{j}^{\Delta M}$ such that its cumulative distribution function equals 0.16 and 0.84 at the lower and upper errors given in table 1 , respectively. ${ }^{10}$

In our signal+background fit, we fix most of the 2 FGL sources to their values from the above background-only fit; exceptions are the sources 2FGL J1037.5-2820, 2FGL J0334.33728 and 2FGL J1505.1+0324, which lie close to the A1060, Fornax and NGC5813 cluster positions, respectively. This leaves us for most of the clusters with three free parameters: the signal normalization $\alpha_{\mathrm{DM}}$ and the two normalizations of the diffuse backgrounds. We checked that leaving more 2FGL source parameters free in the fits does not change our results significantly, but increases the computational time considerably. We scan the likelihood function $\mathcal{L}_{j}^{\Delta M}$ as function of $\alpha_{\mathrm{DM}}$ while refitting the remaining free parameters. Upper limits at the $95 \%$ C.L. (99.7\% C.L.) on the dark matter signal can be derived by increasing the signal until $-2 \log \mathcal{L}_{j}^{\Delta M}$ increases by 2.71 (7.55) from its best-fit value. The significance of a signal can be obtained by comparing the likelihood values that are obtained with and without a dark matter signal.

Finally, to combine the statistical power of the different target regions and to reduce the impact of the cluster mass uncertainties, we performed a combined likelihood analysis of all eight clusters simultaneously. In this case, the combined likelihood function $\mathcal{L}_{\text {comb }}$ is defined as the product of the individual likelihood functions, $\mathcal{L}_{\text {comb }}=\Pi_{j} \overline{\mathcal{L}}_{j}$, where $j$ runs over the different galaxy clusters. The only parameter that is bound to be identical for all targets is the dark matter lifetime or annihilation cross-section. ${ }^{11}$

\footnotetext{
${ }^{9}$ This is a realistic assumption, since these contributions to the dark matter signal could be easily mistaken as part of the extragalactic gamma-ray background as determined by the Fermi LAT collaboration [101]. Since we already include a template for this extragalactic flux, a further inclusion would lead to double counting.

${ }^{10}$ Redoing the error analysis that was performed in ref. [70], we found that a log-normal function describes well the posterior probability distribution function (pdf) of the cluster masses (as long as the polytropic index is $\gamma \approx 1)$. Since $J_{\Delta \Omega}^{j} \propto\left(M_{500}\right)^{\alpha}$ with $\alpha \approx 1$, the same holds for the posterior pdf of $J_{\Delta \Omega}^{j}$. Assuming a flat prior for the cluster masses, this motivates us to adopt a log-normal function also for the likelihood function of $J_{\Delta \Omega}^{j}$.

${ }^{11}$ Note that the angular distance between the targets A1060 and S636 is only $8^{\circ}$ and hence their target regions, but not the signal regions, overlap to a certain degree. We checked that when profiling over the signal
} 
Note that we use our own software to profile over the combined likelihood function in presence of cluster mass uncertainties. These scanning routines were implemented on top of the Fermi Science Tools (and are independent of the routines used in the combined dwarf analysis of ref. [11]).

\section{Limits from the EGBG}

In case of dark matter decay, an important contribution to the gamma-ray signal always comes from our own Galaxy. Assuming an NFW profile $\left(r_{s}=20 \mathrm{kpc}\right.$ and $\left.\rho_{\odot}=0.4 \mathrm{GeV} / \mathrm{cm}^{3}\right)$, we obtain $J_{|b|>10^{\circ}}^{\text {dec. }}=2.1 \cdot 10^{22} \mathrm{GeV} \mathrm{cm}^{-2} \mathrm{sr}^{-1}$ when averaging over the whole sky excluding the Galactic disk, and $J_{\ell=180^{\circ}}^{\text {dec. }}=1.1 \cdot 10^{22} \mathrm{GeV} \mathrm{cm}^{-2} \mathrm{sr}^{-1}$ at the Galactic anti-center (the maximal isotropic component of the Galactic flux). In figure 1 we compare the angular profiles of the cluster decay signal with the contribution from our Galaxy in anti-center direction. As evident from this plot, the Galactic component dominates the signal already at a distance above $\sim 0.5^{\circ}$ from the cluster center.

For comparison with our galaxy cluster limits, we will derive additional limits on decaying dark matter by requiring that the isotropic component of the Galactic signal plus the spatially averaged extragalactic signal does not overshoot the extragalactic gamma-ray background (EGBG) as derived by the Fermi LAT collaboration [101] (see e.g. refs. [33, 102-106]). In the calculation of the prompt signal component, we fully take into account the Galactic (in anti-center direction) and the red-shifted extragalactic signal flux, and we employ for completeness the inter-galactic background light model of ref. [107] for modeling absorption effects. However, our limits do not depend much on the adopted background light model, ${ }^{12}$ since they are dominated by the Galactic signal in most cases (for details of the calculation see ref. [109]). When calculating the ICS component, however, we conservatively only include the extragalactic part, coming from electrons/positrons from dark matter decay that scatters on the CMB. The calculation of the ICS emission inside our Galactic diffusion zone is plagued with uncertainties and a detailed study is beyond the scope of this paper (see e.g. ref. [106] for a thorough discussion). When quoting limits, we will require that in none of the energy bins considered in ref. [101] the dark matter signal integrated over these bins exceeds the measured flux by more than $2 \sigma$. Such limits can be further improved by performing spectral fits [110] or subtracting known astrophysical contributions to the extragalactic gamma-ray background $[111,112]$.

\section{Results}

In none of the galaxy clusters a gamma-ray emission was found at the $3 \sigma$ level, neither when searching for decay nor for annihilation signals with or without substructure contributions. ${ }^{13}$ We derived 95\% C.L. limits on the dark matter lifetime and annihilation cross-section, from individual clusters as well as in a combined likelihood analysis; our results are shown in figures 4, 5, 6 and 7, assuming $100 \%$ branching ratios into $b \bar{b}, \mu^{+} \mu^{-}, W^{+} W^{-}$or $\tau^{+} \tau^{-}$final states.

normalization up to the $2 \sigma$ limits (in order to obtain $\overline{\mathcal{L}}_{j}$ ) the background normalizations are only affected at the $<1 \%$ level, hence the limits on A1060 and S636 remain practically statistically decoupled and the combined likelihood analysis is applicable.

${ }^{12}$ The adopted background light model appears to be in conflict with recent Fermi LAT observations, see ref. [108].

${ }^{13}$ The best signal candidate comes from A1367 (annihilation into $\tau^{+} \tau^{-}, m_{\mathrm{DM}}=10 \mathrm{GeV}$ ) with a trialcorrected significance of $2.7 \sigma$. 

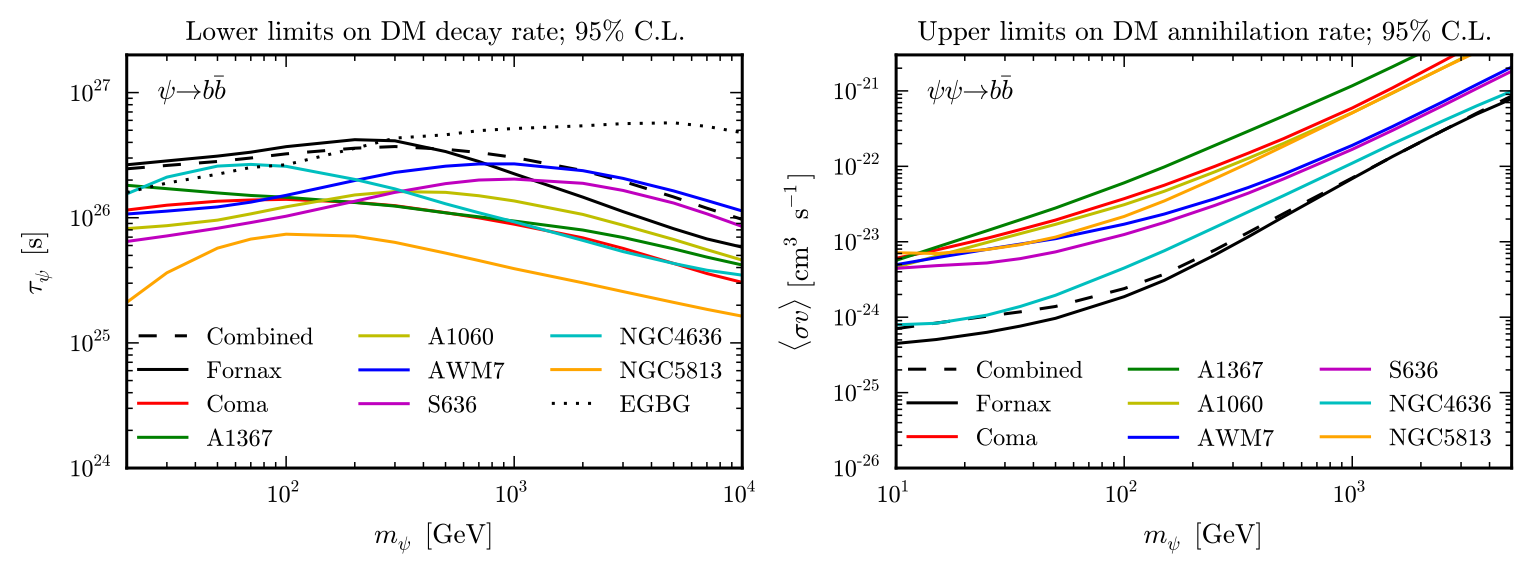

Figure 4. Left panel: lower limits on dark matter lifetime for decay into $b \bar{b}$ final states, as function of the dark matter mass. Solid lines show individual cluster limits, the dashed line the limits from the combined likelihood analysis. The dotted line shows for comparison the limit that can be derived from the EGBG. Right panel: like left panel, but upper limits on annihilation cross-section. Contributions to the signal from dark matter substructures are neglected. Note that the combined limits are sometimes weaker than the strongest individual limits; this is due to a weak preference of a non-zero signal for some of the clusters, see discussion in text.

Main results. For decay or annihilation into $b \bar{b}$, as relevant for MSSM neutralino DM, the strongest individual limits come from the Fornax cluster in most cases, as shown in figure 4 (the impact of dark matter substructures on the annihilation limits is discussed below). Depending on the dark matter mass, lifetimes up to $4 \cdot 10^{26} \mathrm{~s}$ and annihilation cross-sections down to $5 \cdot 10^{-25} \mathrm{~cm}^{3} \mathrm{~s}^{-1}$ can be constrained. Further strong limits come from AWM7, S636 and NGC4636. Our limits on the dark matter lifetime are somewhat weaker than previous results [27]; the difference can be mainly attributed to the fact that we modeled the cluster emission as an extended signal rather than as a point-like source, as we will discuss below.

The limits obtained from our combined likelihood analysis are shown as dashed black lines in figure 4: they are often slightly weaker than the strongest individual limits. This is due to a weak preference for a non-zero signal in some of the clusters (like in A1367). In any case the combined likelihood limit is more robust with respect to uncertainties of the cluster masses, the background modeling and statistical fluctuations in the data than the individual limits (see below discussion and figure 9).

For comparison, the dotted line in the left panel of figure 4 shows the lifetime limits that we obtain from conservatively requiring that the isotropic dark matter signal does not overshoot the EGBG as determined by Fermi LAT [101], see section 3. The EGBG limit clearly dominates the cluster limits for large dark matter masses, whereas the cluster limits are competitive for masses below a few hundred GeV. In any case, since the systematics related to background subtractions are different for EGBG and cluster limits, the limits should be considered as being complementary.

Limits on decay or annihilation into $\mu^{-} \mu^{+}$final states are shown in figure 5 . This channel is relevant for leptophilic models [57-63], that aim to explain the PAMELA/Fermi $e^{ \pm}$anomalies, the corresponding best fit regions being shown in green (PAMELA only) and blue (PAMELA + Fermi + H.E.S.S.) [65]. ${ }^{14}$ In the presented dark matter mass range, the

\footnotetext{
${ }^{14}$ Recently released Fermi LAT results [113] indicate that the positron fraction continues to rise up to energies of $200 \mathrm{GeV}$, which will presumably shift the prefered DM mass range to somewhat higher values.
} 

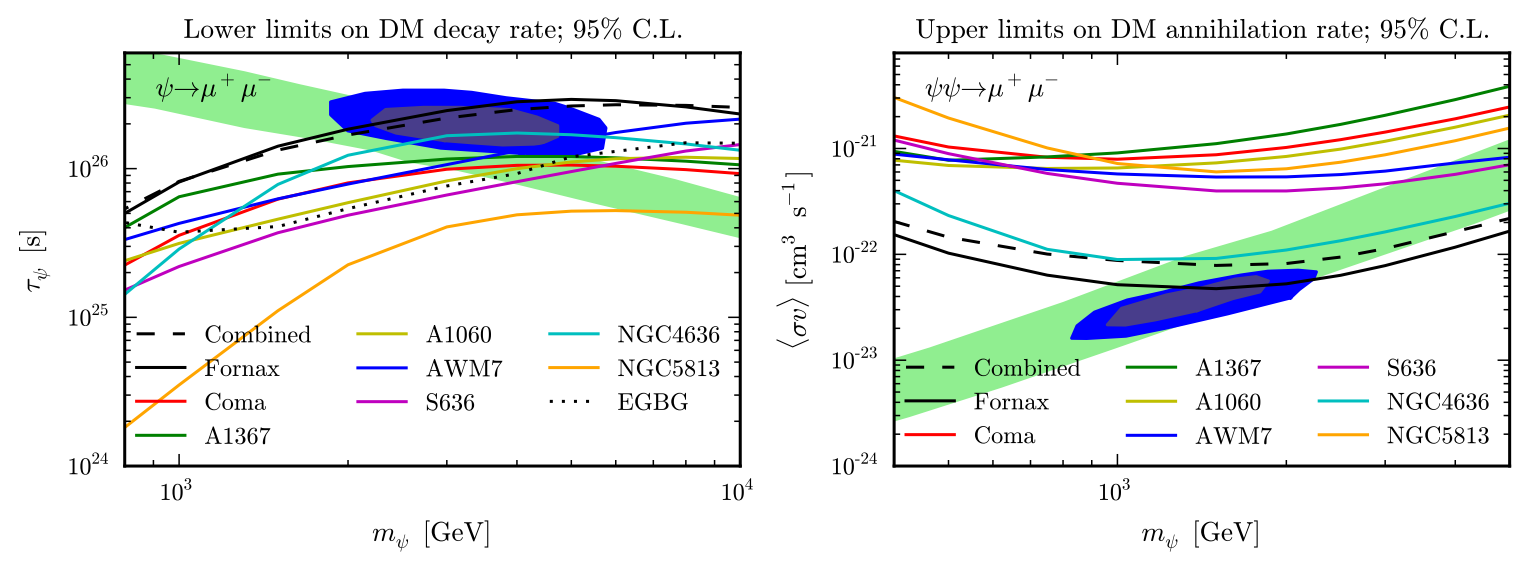

Figure 5. Like figure 4, but for $\mu^{-} \mu^{+}$final states. The blue region indicates the parameter region where a good fit to the PAMELA/Fermi LAT and H.E.S.S. electron/positron data is achieved [65], the green region corresponds to a fit to the PAMELA data only.

dark matter signal is dominated by ICS radiation of the produced electrons and positrons on the CMB; the prompt final-state-radiation can be neglected. Our dark matter lifetime limits reach up to $3 \cdot 10^{26} \mathrm{~s}$ for individual clusters as well as in the combined likelihood analysis, with the strongest limit coming from Fornax. In the case of dark matter annihilation limits down to $6 \times 10^{-23} \mathrm{~cm}^{3} \mathrm{~s}^{-1}$ are obtained. The parameter space favored by PAMELA/Fermi is constrained but not excluded in case of dark matter decay, and remains practically unconstrained in case of dark matter annihilation.

The dotted line in the left panel of figure 5 shows again the limit obtained from the EGBG. In the case of decay into $\mu^{+} \mu^{-}$, the cluster lifetime limits actually dominate over our conservative EGBG limit at all considered dark matter masses. This is due to the fact that we neglected the Galactic ICS emission when calculating the EGBG limit. Such a calculation would require a treatment of cosmic-ray propagation in our Galaxy, which has its own specific uncertainties and is beyond the scope of this paper (see e.g. ref. [106]). On the other hand, as discussed above, propagation effects in galaxy clusters can be neglected at angular scales relevant for LAT observations, making the cluster limits on $\mu^{+} \mu^{-}$final states practically independent of propagation model uncertainties.

In figure 6 , we finally present a summary of our combined likelihood limits on dark matter decay and annihilation into different final states, $b \bar{b}, W^{+} W^{-}$and $\tau^{+} \tau^{-}$. For comparison, we plot the $95 \%$ C.L. as well as the $99.7 \%$ C.L. limits. Limits on $W^{+} W^{-}$are up to massindependent rescaling similar to the limits on $b \bar{b}$; the limits on $\tau^{+} \tau^{-}$clearly indicate that at dark matter masses of $\sim 1 \mathrm{TeV}$ the ICS part of the dark matter signal starts to dominate inside the considered gamma-ray energy range.

Dark matter substructures. In the above limits we neglected contributions from dark matter substructures to the dark matter annihilation signal, firstly to obtain very conservative limits and secondly for the sake of comparison with previous work. However, dark matter substructures are a prediction of cold dark matter scenarios and expected to boost the annihilation signal considerably $[29,30,93,94,96]$. To study their possible impact on our limits, we follow the prescription presented in ref. [30], which builds on results from the Aquarius project $[92,93]$ and leaves the free streaming mass scale $M_{\text {lim }}$ as the only free parameter (see section 2.3 for a discussion). The resulting signal profiles are plotted in the right panel of 

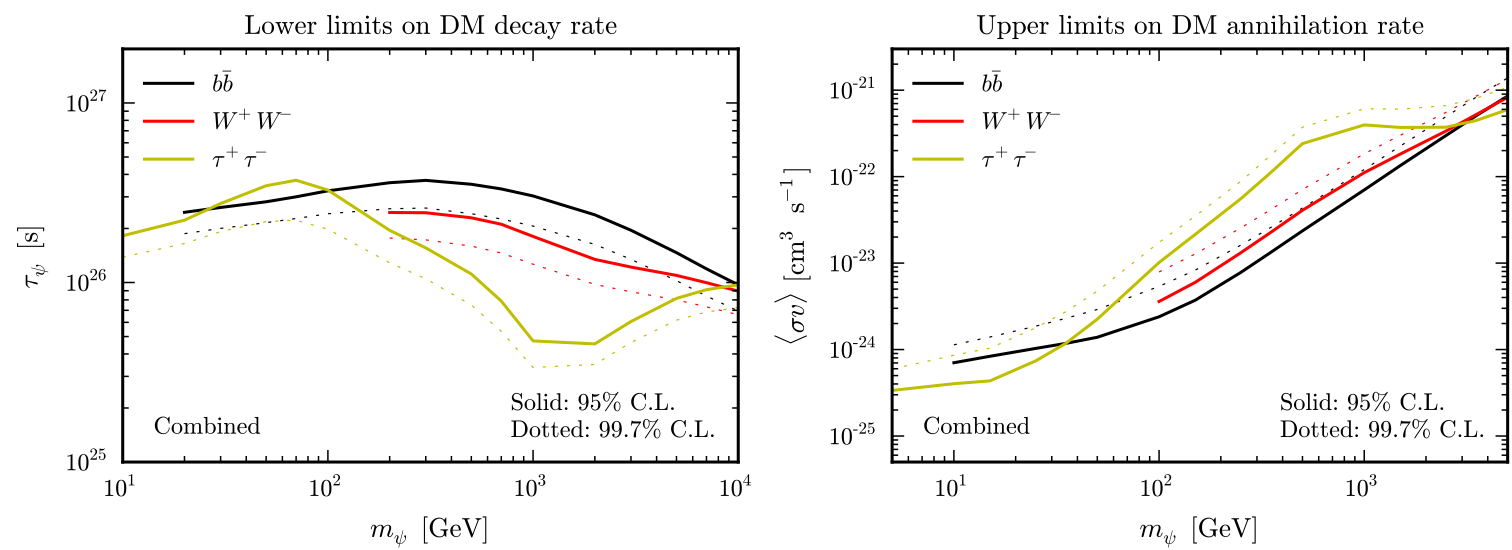

Figure 6. Left panel: lower limits on dark matter decay rate from the combined likelihood analysis, for different final states, as function of the dark matter mass. We show limits at 95\% C.L. (solid) and 99.7\% C.L. (dotted) for comparison. Right panel: corresponding upper limits on annihilation rate. Contributions to the signal from dark matter substructures are neglected in this plot.
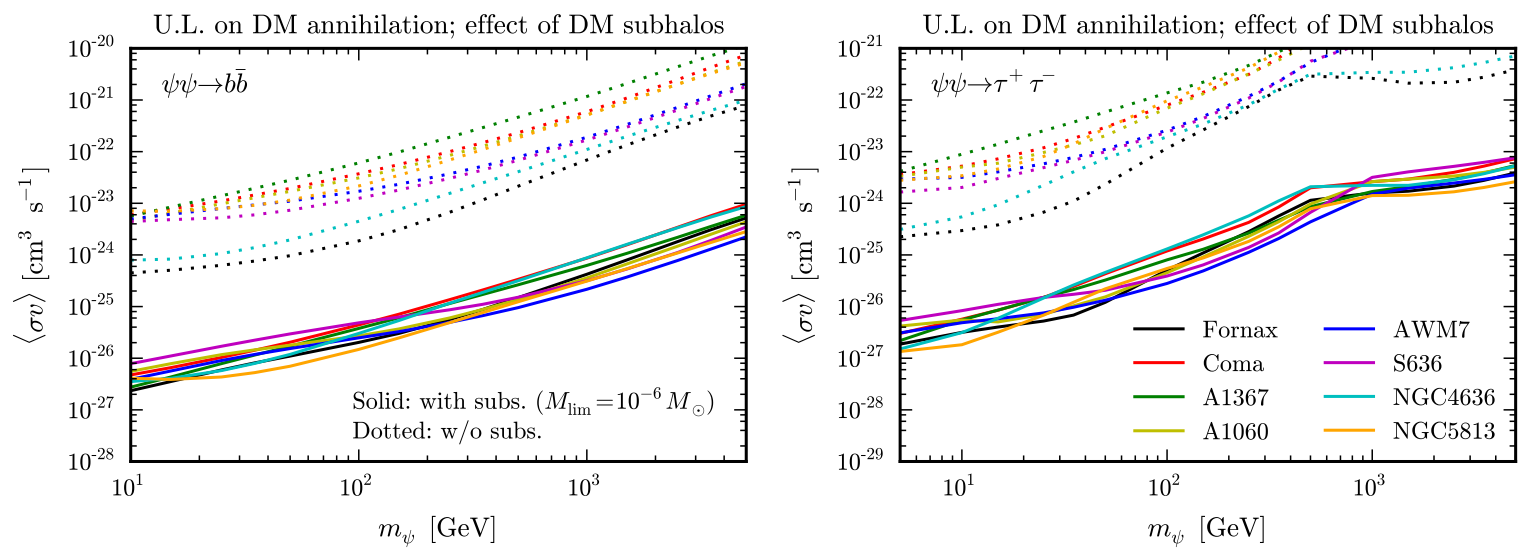

Figure 7. Left panel: individual upper limits on annihilation rate into $b \bar{b}$, including a signal boost from dark matter substructures as discussed in section 2.3 (solid lines). For comparison, the dotted lines show the limits on the unboosted signal, cp. figure 4. Right panel: the same, but for annihilation into $\tau^{+} \tau^{-}$.

figure 1 by the dotted lines, where we adopted a free streaming mass of $M_{\text {lim }}=10^{-6} M_{\odot}$. As can be seen from this plot, the boosted signal profiles extend to much larger radii than the profiles from the smooth dark matter halo alone. The corresponding boost factors of the overall signal are of the order of $10^{3}$, consistent with what is found in refs. [30, 96]. We note, however, that in the recent literature also smaller boost factors for galaxy clusters were discussed $[8,29]$ (see also discussion above), and our adopted boosted fluxes should be considered as being optimistic but not unrealistic.

No evidence for an extended annihilation signal due to dark matter substructures was found. In figure 7 we show the corresponding $95 \%$ C.L. limits on the annihilation cross-section compared to the limits obtained without dark matter substructures taken into account (for simplicity we neglect uncertainties in the overall cluster mass in case of the boosted signal). As expected, we find that the limits are improved by a factor of a few hundred; in the case of dark matter annihilating into $b \bar{b}$ with a thermal cross-section of $3 \times 10^{-26} \mathrm{~cm}^{3} \mathrm{~s}^{-1}$, dark 

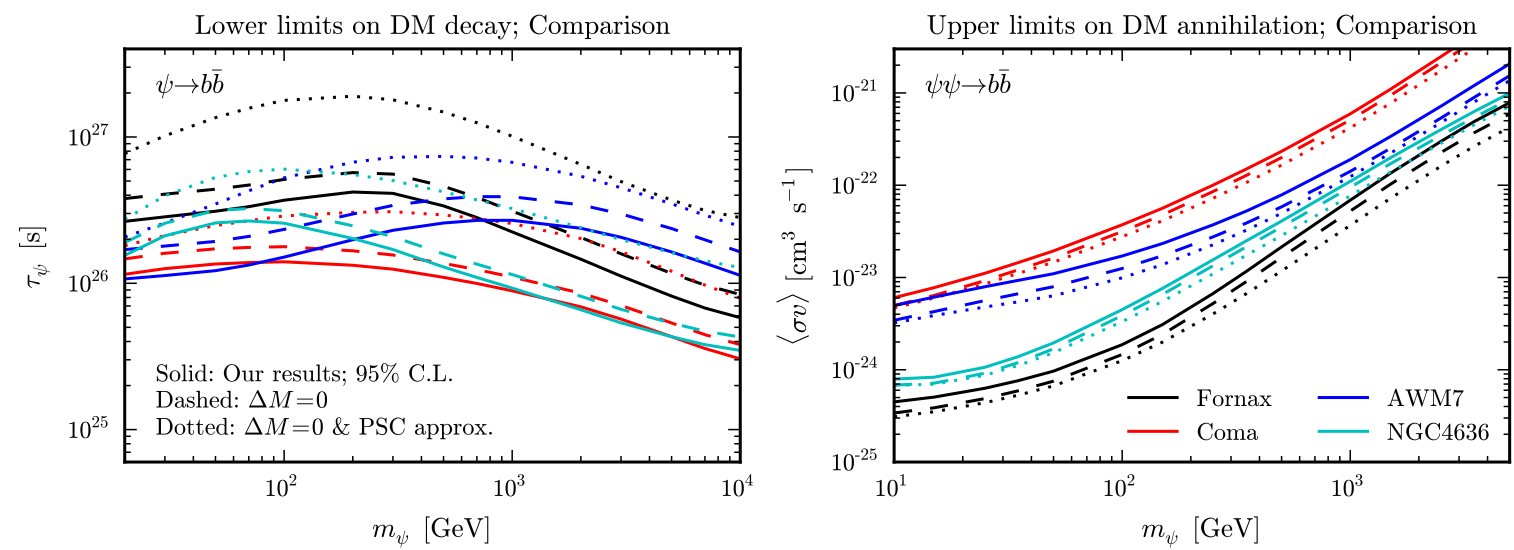

Figure 8. Like figure 4, but comparison of some of our results (solid lines, cp. figure 4) with the limits we would obtain when neglecting cluster mass uncertainties (dashed lines), and when furthermore approximating the cluster emission by a point-like source (dotted lines).

matter masses below $\approx 150 \mathrm{GeV}$ can be excluded. For different values of $M_{\text {lim }}$ the limits would approximately scale like $\propto M_{\mathrm{lim}}^{0.226}$.

Discussion. Comparing our results to previously presented limits [26, 27], we find that despite the increased statistics (at least by a factor of three) our limits on dark matter decay into $b \bar{b}$ remain somewhat weaker than the dark matter lifetime limits presented in ref. [27]. This is due to the point-source approximation of the dark matter signal which was underlying the analysis of ref. [27], as well as the inclusion of cluster mass uncertainties in the present work. In the case of dark matter annihilation our limits improve the results from ref. [26] by up to a factor of two.

In figure 8 we compare our $95 \%$ C.L. limits on decay or annihilation into $b \bar{b}$ (solid lines) with the limits we would obtain when dropping the cluster mass uncertainties (dashed lines), and when furthermore approximating the dark matter signal as a point-like source (dotted lines). In the latter case, the extended signals are integrated over a region with a radius of $1^{\circ}$ and modeled as a point source at the cluster center, following ref. [26, 27]. As can be seen from figure 8 , the non-inclusion of uncertainties in the cluster masses could strengthen the limits by up to $\approx 50 \%$ in some cases, similar for both dark matter decay and annihilation. A point-source approximation to the signal from decaying dark matter would lead to a further strengthening of the corresponding limits by a factor of a few in some cases, as shown in the left panel of figure 8 (a similar impact is expected in case of annihilation signals boosted by dark matter substructures). However, in case of an unboosted annihilation signal (right panel), the point-source approximation to the signal changes the limits only on the $10 \%-30 \%$ level and hence appears to be justified, in agreement with what was found in ref. [26].

The robustness of a limit with respect to the underlying statistics and uncertainties can be inferred from its dependence on the adopted C.L. In figure 9 we show for the case of dark matter decay into $b \bar{b}$ our individual and combined limits at 95\%, 99.7\% and 99.994\% C.L. in comparison. As can be seen from these plots, the combined limit depends considerably less on the adopted C.L. than the individual limits. This is due to the fact that uncertainties related to the cluster mass, as well as statistical fluctuations in the target region, tend to average out in the combined likelihood analysis. Furthermore, note that systematic effects in the modeling of the astrophysical backgrounds become in general less important at higher C.L. 


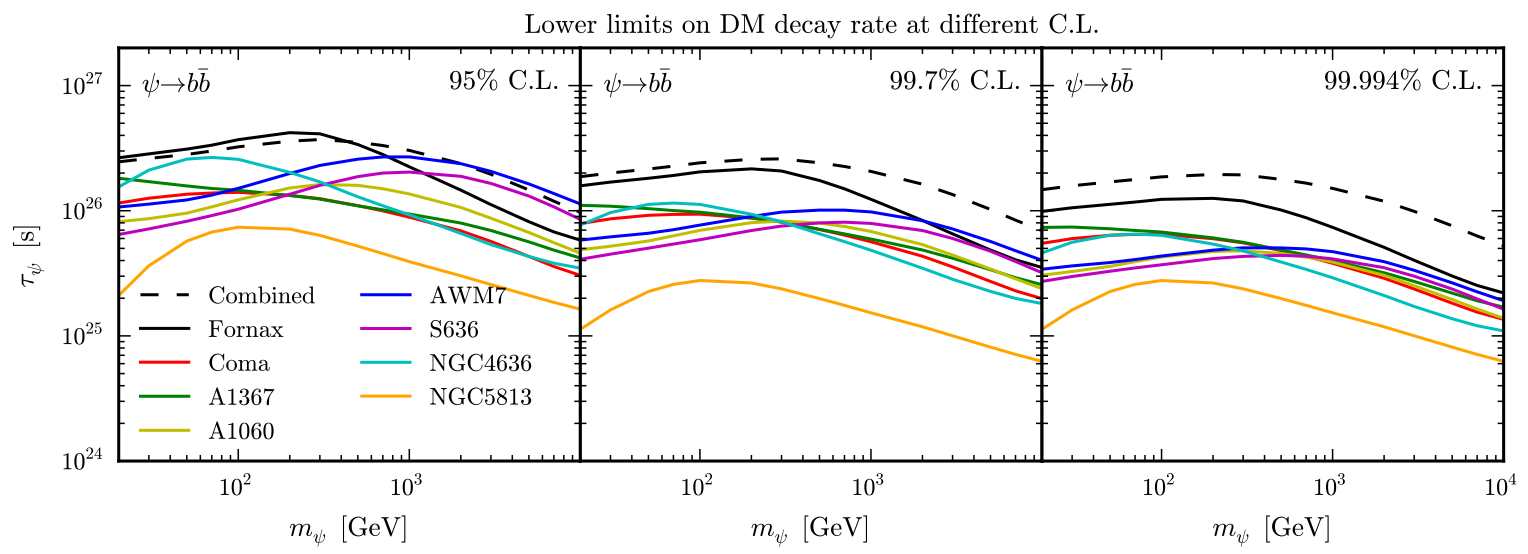

Figure 9. Like left panel of figure 4, but at different C.L. Note that the combined limit is less dependent on the C.L. than the individual limits, since uncertainties in the cluster masses tend to average out if limits are combined.

In this paper we adopted galaxy cluster masses that are based on the X-ray observations from refs. [69, 70], the HIFLUGCS catalog. The main advantage of this catalog is its completeness. Many other X-ray measurements of cluster masses exist (see e.g. refs. [114-116]), which are however often concentrating on only one single cluster at a time, and hence difficult to use in a combined analysis. Important assumptions that enter the mass determination via $\mathrm{X}$-ray observations is that the intra-cluster gas is in hydrostatic equilibrium and spherically distributed; further assumptions concern the temperature gradient of the gas, which is often approximated to be zero, as well as the radial distribution of the gas density. The systematical errors made by these approximations are very difficult to estimate, and it is likely that a neglect leads to a too optimistic determination of the cluster mass uncertainties.

Fortunately, X-ray measurements are not the only way to determine the mass of a galaxy cluster; other methods include studies of the velocity dispersion of cluster member galaxies and weak gravitational lensing (see e.g. refs. [117-120]). A comparison of the masses derived from different methods can be used as a rough estimate for the overall systematic errors in the cluster mass measurements. In refs. [26] and [27], the dark matter signal fluxes that follow from different cluster mass measurements (namely for the M49, Fornax and Coma clusters) were compared, and it was concluded that the overall uncertainties are roughly of the order of a factor of $\sim 2$ and not systematically biased with respect to the HIFLUGCS X-ray values. Looking at table 1 , one can see that this is somewhat larger than what follows from the mass uncertainties given in the HIFLUGCS catalog; the latter are derived from uncertainties in X-ray profile fit, the temperature measurements and the temperature gradient. Hence, we expect that our limits on the dark matter annihilation cross section and lifetimes are not the most conservative ones that one could obtain for individual clusters when exploiting all systematic uncertainties. The advantage of our combined analysis is that, in absence of a systematic bias of the HIFLUGCS catalog, these kind of uncertainties are expected to partially average out.

In the above calculation of the dark matter induced ICS emission the possible impact of intra-cluster magnetic fields was neglected; this is only justified if the magnetic fields remain well below the critical value of $B_{\mathrm{CMB}}=3.2 \mu \mathrm{G}$. However, since Faraday rotation based measurements in galaxy clusters find magnetic fields of a few $\mu \mathrm{G}$ (see e.g. [78, 121, 122]), 
the validity of this approximation is not guaranteed. For most of our targets in table 1 the magnetic fields are not precisely know, which makes it difficult to systematically include their effects. However, in case of the very massive Coma cluster the magnetic field was studied in ref. [121], and a model for the magnetic field profile was presented. Adopting this model we can calculate how our limits change when synchrotron emission of the electrons and positrons produced in the dark matter decay or annihilation is included. We find that in case of the Coma cluster the limits on dark matter annihilation into $\mu^{+} \mu^{-}$as shown in figure 5 are weakened by a factor of around two, whereas the effect on decaying dark matter limits is negligible (the same is true for the extended annihilation signals coming from dark matter substructures). In the adopted magnetic field model, the field exceeds the critical value $B_{\mathrm{CMB}}$ only close to the cluster center at angles $\theta \lesssim 0.17^{\circ}$, making the impact on point-source like signals large and on extended signals small. We conclude that intra-cluster magnetic fields are unlikely to affect our decaying dark matter limits or the limits on subhalo-boosted annihilation signals.

\section{Consequences for gravitino dark matter}

The gravitino is the spin-3/2 supersymmetric partner of the graviton. If the lightest superparticle (LSP), it provides a natural dark matter candidate [123], the mass of which can vary from $m_{3 / 2} \sim \mathrm{eV}$ to $\sim \mathrm{TeV}$ depending on the details of the supersymmetry breaking mechanism. Gravitinos are produced in the early universe through 2-to-2 thermal scatterings with an abundance which is proportional to the reheating temperature $T_{R}$ after inflation

$$
\Omega_{3 / 2}^{\text {th }} h^{2}=C\left(\frac{100 \mathrm{GeV}}{m_{3 / 2}}\right)\left(\frac{m_{\tilde{g}}}{1 \mathrm{TeV}}\right)^{2}\left(\frac{T_{R}}{10^{10} \mathrm{GeV}}\right)
$$

where $m_{3 / 2}$ and $m_{\tilde{g}}$ are the gravitino and gluino masses respectively, and $C \simeq 0.5$ to leading order in the gauge couplings $[124-126]{ }^{15}$ In addition, gravitinos may also be produced through the gravitational decay of the NLSP. However, for $\Omega_{\mathrm{NLSP}} h^{2} \ll 1$ or $m_{\mathrm{NLSP}} \gg m_{3 / 2}$ the latter contribution is negligible [127]. Moreover, inflaton decay may also contribute to the production mechanism [128]. In what follows, thermal leptogenesis is assumed to be responsible for the generation of the observed baryon asymmetry. In such a case, high reheating temperatures are required, and the dominant gravitino production mechanism is the thermal one. Particularly, for $T_{R} \sim 10^{10} \mathrm{GeV}[129,130]$ a gravitino abundance of the order of the observed dark matter relic density $\Omega_{\mathrm{DM}}=0.11[72]$ is achieved for typical supersymmetric parameters, i.e. $m_{3 / 2} \sim 100 \mathrm{GeV}$ and $m_{\tilde{g}} \sim 1 \mathrm{TeV}$. However, as it is well known, such high values of the gravitino mass lead to slow NLSP decays and can dramatically affect the successful predictions of the standard big bang nucleosynthesis (BBN) scenario [131-137].

Among the different scenarios proposed to reconcile thermal leptogenesis, gravitino dark matter and BBN, a mild violation of $R$-parity inducing a rapid decay of the NLSP before the onset of the BBN is of interest [31]. In such a case, the gravitino is not stable anymore, but still provides a viable dark matter candidate due to the double suppression of its decay, by the Planck scale as well as by the small $R$-parity breaking parameter. Interestingly, this opens up the way to look for traces of gravitino decays in cosmic-ray fluxes, such as antimatter $[36,37]$ and neutrino [39]. Additionally to the intense gamma-ray line arising from

\footnotetext{
${ }^{15}$ Note that $C$ has $\mathcal{O}(1)$ uncertainty due to unknown higher order contributions and nonperturbative effects [124].
} 
the $\psi_{3 / 2} \rightarrow \gamma \nu$ two-body decay [31-35, 37], the produced gamma-ray flux typically features a continuous component generated by the fragmentation of the Higgs and gauge bosons.

In what follows, we apply the above analysis to the decaying gravitino scenario. Contrarily to gamma-ray lines, galaxy clusters offer more sensitivity to large gravitino masses, thus rendering the present analysis supplementary to our previous gamma-ray lines study [47]. Following the structure of the latter, we first summarize the bilinear $R$-parity violation supersymmetric framework considered here. We then present limits on the size of $R$-parity violation and finally discuss the prospect for seeing long-lived neutralino and stau NLSPs at the LHC.

\section{$5.1 \quad R$-parity breaking model}

The supersymmetric standard model with explicit bilinear $R$-parity violation is specified by the superpotential

$$
W=W_{\mathrm{MSSM}}+\mu_{i} H_{u} l_{i},
$$

as well as by the soft supersymmetry breaking potential

$$
\mathcal{L}=\mathcal{L}_{\text {soft }}^{\mathrm{MSSM}}+B_{i} H_{u} \tilde{l}_{i}+m_{i d}^{2} \tilde{l}_{i}^{\dagger} H_{d}+\text { h.c. },
$$

where $W_{\text {MSSM }}$ and $\mathcal{L}_{\text {soft }}^{\mathrm{MSSM}}$ are the $R$-parity conserving MSSM superpotential and scalar Lagrangian, $H_{u / d}$ are the up/down-type Higgs doublets, $l_{i}$ the lepton doublets, and $\mu_{i}, B_{i}$ and $m_{i d}^{2}$ are the $R$-parity violating couplings. Trading the mass mixing parameters for $R$ parity breaking Yukawa couplings as proposed in ref. [138], the gravitino decay is function of a single dimensionless parameter $\zeta$, which also enters the decay of the NLSPs of interest (see ref. [138] for a definition of $\zeta$ in terms of the bilinear $R$-parity violating couplings $\mu_{i}, B_{i}$ and $\left.m_{i d}^{2}\right)$.

Two typical sets of boundary conditions for the supersymmetry breaking parameters of the MSSM at the grand unification (GUT) scale are investigated in the following, resulting in two different types of NLSPs. First we consider equal scalar and gaugino masses

$$
\text { (A) } \quad m_{0}=m_{1 / 2}, \quad a_{0}=0, \quad \tan \beta=10,
$$

for which the bino-like neutralino $\widetilde{\chi}_{1}^{0}$ is the NLSP. In the second one, which corresponds to no-scale models or gaugino mediation,

$$
m_{0}=0, \quad m_{1 / 2} \neq 0, \quad a_{0}=0, \quad \tan \beta=10,
$$

the lightest stau $\tilde{\tau}_{1}$ is the NLSP. In both cases, $\tan \beta=10$ has been chosen as a representative value, and the trilinear scalar coupling $a_{0}$ has been set to zero for simplicity. For both sets of boundary conditions, the universal gaugino mass $m_{1 / 2}$ remains as the only independent variable, and the gaugino masses $M_{1,2,3}$ satisfy the following relations at the electroweak scale

$$
\frac{M_{3}}{M_{1}} \simeq 5.9, \quad \frac{M_{2}}{M_{1}} \simeq 1.9 .
$$

Electroweak precision tests (EWPT) yield important lower bounds on the superparticle mass spectrum [126]. For a neutralino NLSP, the most stringent constraint comes from the Higgs potential. The universal gaugino mass $m_{1 / 2}$ is required to be high enough in order for the Higgs mass to fulfills the LEP lower bound $m_{h}>114.4 \mathrm{GeV}$ [139]. This implies the 
lower limit $m_{\chi_{1}^{0}} \gtrsim 130 \mathrm{GeV} .{ }^{16}$ However, allowing negative $a_{0}$ or scalar masses much larger than $m_{1 / 2}$ at the GUT scale would weaken this limit, and we will take $m_{\chi_{1}^{0}}>100 \mathrm{GeV}$ as a lower bound for the neutralino mass subsequently. In the stau NLSP case, the lower bound comes from the absence of pair production of heavy charged particles at LEP and reads $m_{\tilde{\tau}_{1}}>100 \mathrm{GeV}$ [139]. Rewriting eq. (5.1)

$$
m_{\mathrm{NLSP}} \simeq 310 \mathrm{GeV}\left(\frac{\xi}{0.2}\right)\left(\frac{m_{3 / 2}}{100 \mathrm{GeV}}\right)^{1 / 2}\left(\frac{10^{9} \mathrm{GeV}}{T_{R}}\right)^{1 / 2},
$$

where $\xi \equiv m_{\mathrm{NLSP}} / m_{\tilde{g}}$ is implicitly fixed by the supersymmetry breaking boundary conditions [126], we get absolute upper bounds on the NLSP masses requiring the gravitino to be the LSP. In the case of the neutralino NLSP, eq. (5.7) implies $m_{\chi_{1}^{0}} \lesssim 690 \mathrm{GeV}$ for $\xi=1 / 5.9$, and is essentially independent of $m_{0}$ and $\tan \beta$. For the stau NLSP, $\tan \beta=10$ yields $\xi=1 / 6.2$, which consequently leads to the more stringent bounds $m_{\tilde{\tau}_{1}} \lesssim 615 \mathrm{GeV}$. Note that there is a strong dependence on $\tan \beta$ in that case [126], and that $\xi$ decreases with increasing $\tan \beta$.

For a typical effective neutrino mass $\widetilde{m}_{1}=10^{-3} \mathrm{eV}$, successful thermal leptogenesis requires a minimal reheating temperature of $T_{R} \sim 10^{9} \mathrm{GeV}$ [130]. Using eq. (5.1) together with a lower bound on the gluino mass $m_{\tilde{g}} \gtrsim 815 \mathrm{GeV}$ [140], this implies a lower bound for the gravitino mass $m_{3 / 2} \gtrsim 30 \mathrm{GeV}$.

\subsection{Limits from galaxy clusters}

The gamma-ray spectrum produced through gravitino decays features two types of contributions: first, the $\psi_{3 / 2} \rightarrow \gamma \nu$ two body decay produces a gamma-ray line, a channel which is dominant below the $W$ threshold. ${ }^{17}$ Additionally, both the fragmentation of the Higgs and gauge bosons as in $Z^{0} \nu$ and $h^{0} \nu$ final states, as well as the final state radiation of the charged leptons produced in the $W^{ \pm} l^{\mp}$ final states, generate a continuum spectrum. The relative strength of these two is fixed by the corresponding branching ratios, that we present in figure 10 together with representative spectra following refs. $[37,39] .{ }^{18}$ While the search for gamma-ray lines of galactic origin through deviations from a power law background is efficient for contained continuum contributions [47], i.e. for $m_{3 / 2} \lesssim 200-300 \mathrm{GeV}$, constraints from galaxy clusters observations and the EGBG dominates the gamma-ray line ones for $m_{3 / 2} \gtrsim 250 \mathrm{GeV}$, as illustrated in figure 11 . This agreeably makes gamma-ray line searches, galaxy cluster observations and EGBG studies complementary. As a result the gravitino lifetime is constrained to be at least $\tau_{3 / 2} \gtrsim \mathcal{O}\left(10^{26} \mathrm{~s}\right)$ in all the gravitino mass range considered. Subsequently, we will concentrate on the limits derived by galaxy cluster observations, and leave a detailed study of implications from the EGBG to future work.

\subsubsection{R-parity breaking parameter}

The gravitino inverse decay rate into photon/neutrino pairs is given by [32, 138]

$$
\Gamma_{\psi_{3 / 2} \rightarrow \gamma \nu}^{-1}=\frac{32 \sqrt{2}}{\alpha \zeta^{2}} \frac{G_{\mathrm{F}} M_{\mathrm{P}}^{2}}{m_{3 / 2}^{3}} \frac{M_{1}^{2} M_{2}^{2}}{\left(M_{2}-M_{1}\right)^{2}}\left(1+\mathcal{O}\left(s_{2 \beta} \frac{m_{Z}^{2}}{\mu^{2}}\right)\right),
$$

\footnotetext{
${ }^{16}$ Note that $m_{\chi_{1}^{0}} \simeq M_{1}$ with good accuracy [138].

${ }^{17}$ Note that three-body decays with intermediate massive gauge bosons are expected to contribute by more than $10 \%$ below the kinematic threshold [43, 44, 141].

${ }^{18}$ Note that the branching ratio into lines is in principle model-dependent.
} 

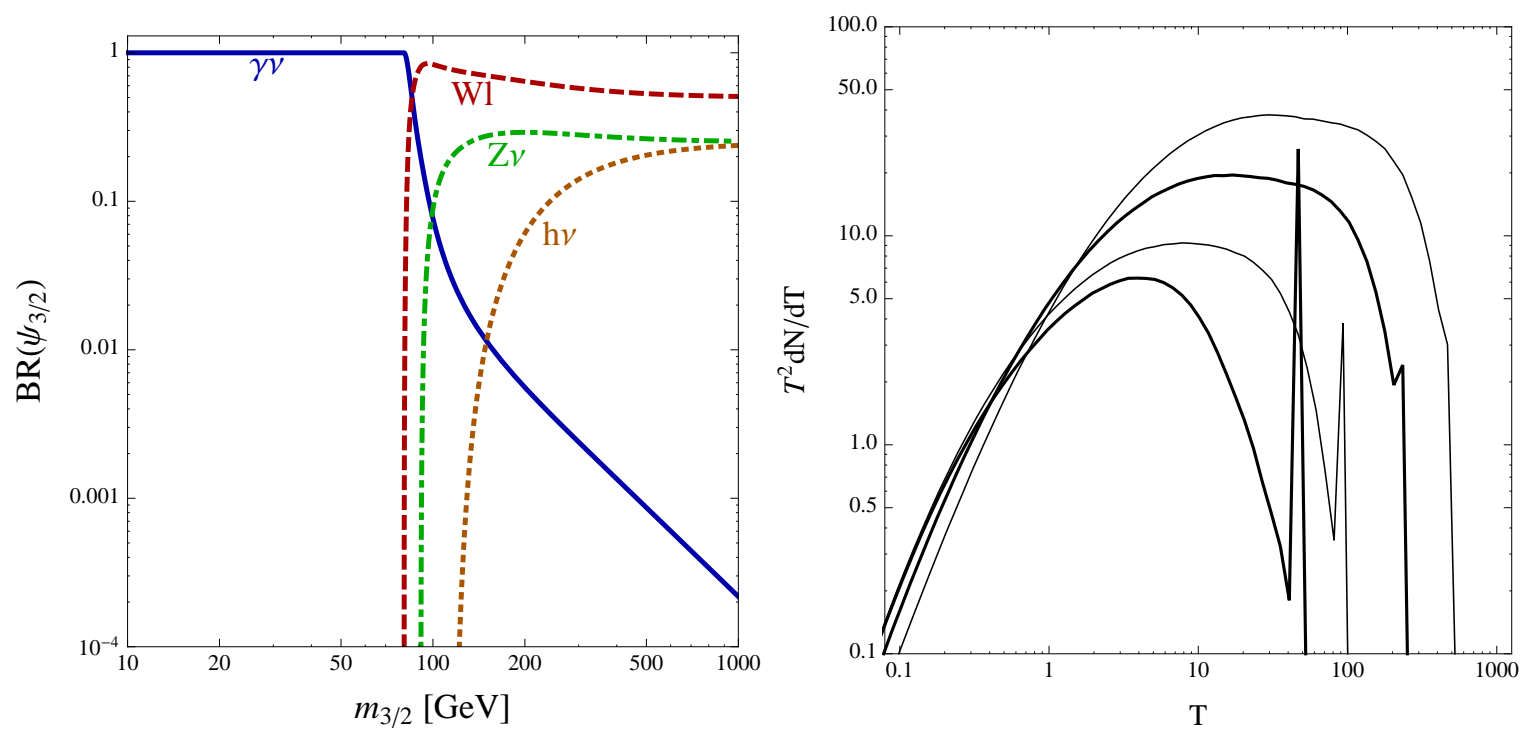

Figure 10. Left: two-body decay branching ratios of the gravitino. Right: gamma-ray spectra for $m_{3 / 2}=100,200,500$ and $1000 \mathrm{GeV}$. We adopt here the same set of parameters as in refs. [37, 39].

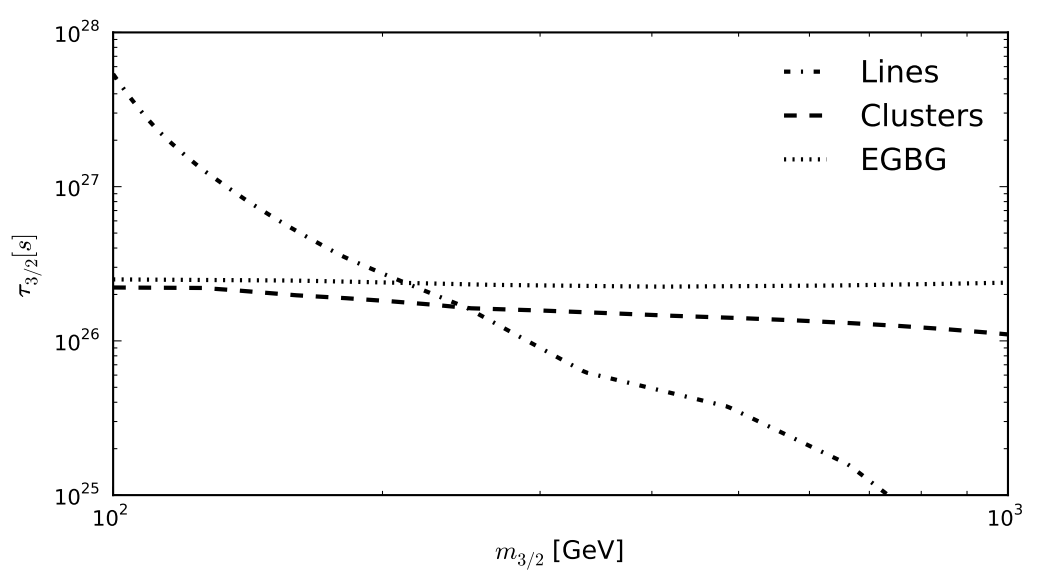

Figure 11. Lower limits on the gravitino lifetime. The dot-dashed line shows the gamma-ray line limits, the dashed line the limits resulting from the combined cluster analysis, and the dotted line the EGBG limits.

where $\alpha$ is the electromagnetic fine structure constant, $M_{\mathrm{P}}=2.4 \times 10^{18} \mathrm{GeV}$ the reduced Planck mass, and $G_{\mathrm{F}}=1.16 \times 10^{-5} \mathrm{GeV}^{-2}$ is the Fermi constant. Using the strongest limits on the total gravitino lifetime illustrated in figure 11 together with the branching ratios presented in figure 10, this expression can be used to derive conservative upper-limits on the $R$-parity breaking parameter $\zeta$. To do so, one has to consider for a given gravitino mass the maximally allowed bino mass which results from the combination of eqs. (5.1) and (5.6) when considering the lowest reheating temperature allowed in the thermal leptogenesis scenario, i.e. $T_{R} \sim 10^{9} \mathrm{GeV}$. The results are presented in figure 12. Note that at high gravitino masses, the production of anti-protons in Higgs and gauge bosons fragmentation could further constrain the $\zeta$ parameter. 


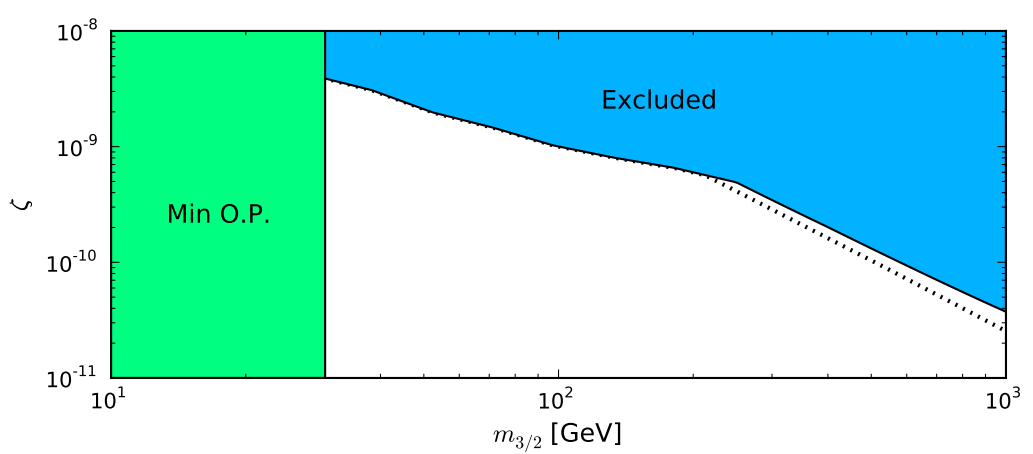

Figure 12. Upper bounds on the $R$-parity violation parameter $\zeta$, derived from the Fermi LAT limits in figure 11. For thermal leptogenesis, overproduction (O.P.) of gravitinos already excludes the left green region. The dotted line illustrates the limits from EGBG.

\subsubsection{Stau NLSP decay length}

In the case of the $\tilde{\tau}_{1}$-NLSP, the total decay width of the lightest mass eigenstate is a mixture of left and right handed partial decays

$$
\Gamma_{\tilde{\tau}_{1}}(\epsilon)=\sin ^{2} \theta_{\tilde{\tau}} \Gamma_{\tilde{\tau}_{L}}(\epsilon)+\cos ^{2} \theta_{\tilde{\tau}} \Gamma_{\tilde{\tau}_{R}}(\epsilon) .
$$

where the dimensionless parameter $\epsilon$ is directly related to the $R$-parity violating Yukawa couplings (see ref. [138] for details). Since the latter are typically proportional to the ordinary Yukawa couplings, decays into second and third families dominate. For definiteness, we will below assume a flavor structure as described in ref. [138], into which the chiral state decays are dominated by the following channels

$$
\begin{aligned}
\tilde{\tau}_{R} & \rightarrow \tau_{L} \nu, \mu_{L} \nu, \\
\tilde{\tau}_{L} & \rightarrow \bar{t}_{R} b_{L} .
\end{aligned}
$$

Assuming $\zeta \simeq \epsilon,^{19}$ and using the cluster upper limits on $\zeta$ from figure 12, we can derive lower bounds on the stau decay length. Our results are shown in figure 13. The parameter space is already constrained by EWPT and overproduction bounds, and the lower limits on the neutralino decay length vary between $100 \mathrm{~m}$ and $10 \mathrm{~km}$. It is interesting that if such particles were to be produced at the LHC, a sizable amount of their decays could take place in the detector $[38,142]$. We obtain a lowest possible decay length $c \tau_{\tilde{\tau}_{1}} \simeq 200 \mathrm{~m}$ for $m_{3 / 2} \simeq 30 \mathrm{GeV}$ and $m_{\tilde{\tau}_{1}} \simeq 130 \mathrm{GeV}$.

\subsubsection{Neutralino NLSP decay length}

A neutralino NLSP heavier than $100 \mathrm{GeV}$ dominantly decays into $W^{ \pm} \ell^{\mp}$ and $Z^{0} \nu[143,144]$. The corresponding decay width is directly proportional to the $R$-parity breaking parameter $\zeta$ squared, which also enters the gravitino decay width eq. (5.8). As a consequence, the two quantities can be related through [138]

$$
\tau_{\tilde{\chi}_{1}^{0}}=\frac{c_{w}^{2}}{2 \sqrt{2}} \frac{\left(M_{2}-M_{1}\right)^{2}}{M_{2}^{2}} \frac{m_{3 / 2}^{3}}{G_{\mathrm{F}} M_{\mathrm{P}}^{2} m_{\chi_{1}^{0}}^{3}} \frac{\Gamma_{\psi_{3 / 2} \rightarrow \gamma \nu}^{-1}}{2 f\left(m_{\chi_{1}^{0}}, m_{W}\right)+f\left(m_{\chi_{1}^{0}}, m_{Z}\right)},
$$

\footnotetext{
${ }^{19} \zeta$ values much smaller than $\epsilon$ can be achieved through a proper choice of the parameters $\mu_{i}, B_{i}$ and $m_{i d}^{2}$.
} 


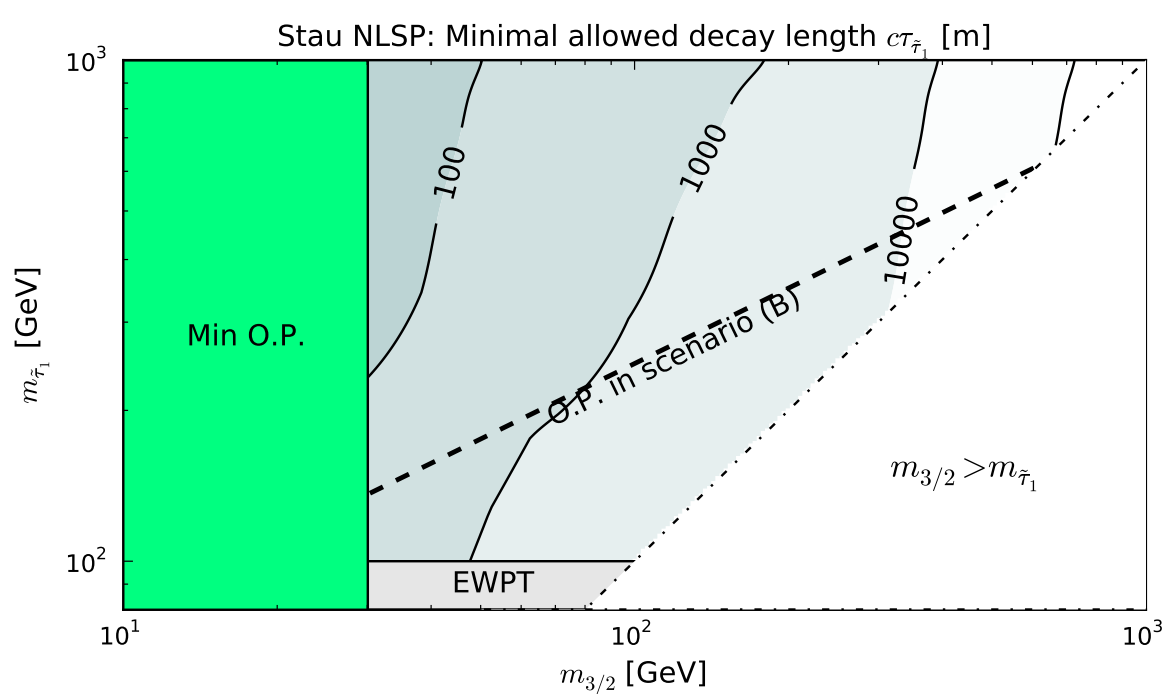

Figure 13. Contour plot of lower bounds on the stau NLSP decay length coming from cluster and gamma-ray line constraints on the gravitino lifetime, as function of the stau and gravitino masses, $m_{\tilde{\tau}_{1}}$ and $m_{3 / 2}$ respectively. The lower gray region is excluded by electroweak precision tests (EWPT). For thermal leptogenesis, overproduction (O.P.) of gravitinos excludes at minimum the left green region, a limit which strengthens to the black-dashed line when assuming the universal boundary conditions (B), cf. eq. (5.5). The lower-right exclusion comes from the gravitino LSP requirement.

where the phase space factor $f$ is defined by

$$
f\left(m_{1}, m_{2}\right)=\left(1-\frac{m_{2}^{2}}{m_{1}^{2}}\right)^{2}\left(1+2 \frac{m_{2}^{2}}{m_{1}^{2}}\right) .
$$

Using the gaugino mass relation eq. (5.6), lower bounds on the neutralino decay length $c \tau_{\tilde{\chi}_{1}^{0}}$ can be derived from the partial gravitino decay width. Our results are summarized in figure 14 considering the cluster lifetime limits. For the parameter space allowed by EWPT and overproduction bounds, we obtain minimal decay lengths $\mathcal{O}(100 \mathrm{~m}-100 \mathrm{~km})$, which are in the range of detectability of the LHC [38, 142]. Decay lengths as small as $c \tau_{\tilde{\chi}_{1}^{0}} \simeq 60 \mathrm{~m}$ are allowed for $m_{3 / 2} \simeq 30 \mathrm{GeV}$ at $m_{\chi_{1}^{0}} \simeq 140 \mathrm{GeV}$.

\section{Conclusions}

Galaxy clusters are the most massive collapsed objects in the Universe, and very interesting targets for the indirect search for dark matter. Using three years of Fermi LAT data, we studied the gamma-ray emission from eight of the most promising galaxy clusters and searched for signatures for dark matter decay or annihilation. We analyzed the clusters individually as well as in a combined likelihood approach. We took into account uncertainties in the cluster masses as determined by ROSAT PSPC X-ray observations and modeled the dark matter signals as extended sources. Our main results are:

- In none of the eight galaxy clusters listed in table 1 a significant gamma-ray emission that could be attributed to dark matter decay or annihilation was found. We derived limits at the $95 \%$ C.L. on the dark matter lifetime and on the annihilation cross-section, from each cluster individually as well as in a combined likelihood approach. Our results 


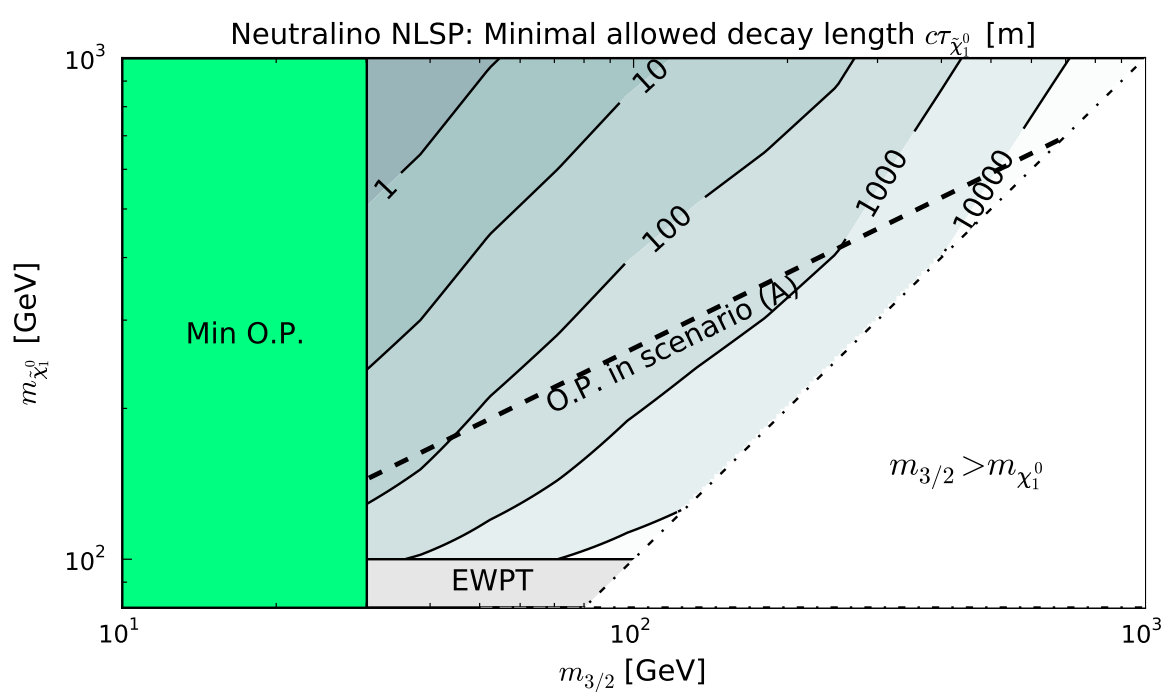

Figure 14. Like figure 13, but for a neutralino NLSP.

are shown in the figures 4, 5, 6 and 7 for $b \bar{b}, \mu^{+} \mu^{-}, W^{+} W^{-}$and $\tau^{+} \tau^{-}$final states. In most cases the combined limits are at the level of the strongest individual limits; in any case the combined limits are more robust with respect to uncertainties of the cluster masses, background modeling and statistical fluctuations in the cluster target regions (figure 9).

- The limits on the dark matter lifetime turn out to be somewhat weaker than what previous results indicated [27], reaching up to lifetimes of $\tau_{\mathrm{DM}} \simeq 4 \times 10^{26} \mathrm{~s}$ in case of decay into $b \bar{b}$ (figure 4 ). The difference can be partly attributed to the fact that we modeled the cluster emission as an extended signal rather than as a point-like source, and partly to the inclusion of cluster mass uncertainties. We find that a point-source approximation to the signal from dark matter decay could in some cases strengthen the limits wrongly by a factor of a few (figure 8). In particular, the decaying dark matter interpretation of the $e^{ \pm}$excess in terms of dark matter decaying into $\mu^{+} \mu^{-}$remains only partially constraint by our galaxy cluster limits (figure 5).

- As long as prompt radiation dominates the overall gamma-ray signal, our cluster limits on the dark matter lifetime are sometimes weaker than the corresponding limits that can be conservatively inferred from measurements of the extragalactic gamma-ray background (EGBG, see figures 4 and 5). However, at lower gamma-ray energies the cluster and EGBG limits are comparable and should be considered as being complementary, since the systematics of the background subtraction are in general very different.

- In case of an annihilation signal coming from the smooth component of the cluster dark matter halo alone, while neglecting the contributions from dark matter substructures, previously presented limits [26] are improved by up to a factor of two (figures 4,5 and 6 ). In case of annihilation into $b \bar{b}$ final states, we obtain limits down to $5 \times 10^{-25} \mathrm{~cm}^{3} \mathrm{~s}^{-1}$ for a $10 \mathrm{GeV}$ WIMP, and we confirm that the point-source approximation to the dark matter emission is valid when the effect of dark matter substructures are neglected. However, as we discuss for the case of the Coma cluster, for such a point-like emission 
intra-cluster magnetic fields could reduce the unboosted annihilation signal by an order one factor when the signal is dominated by photons from ICS radiation.

- When contributions to the annihilation signal from dark matter substructures are taken into account, the dark matter signal must be modeled as an extended source (cp. figure 1). Adopting the optimistic scenario for signal boosts due to substructures from ref. [30], we find that the limits could strengthen by a factor of a few hundred if substructures with masses down to $M_{\lim }=10^{-6} M_{\odot}$ are included. In this case, the limits on $b \bar{b}$ would start to reach the thermal cross-section $3 \times 10^{-26} \mathrm{~cm}^{3} \mathrm{~s}^{-1}$ and exclude dark matter masses below $150 \mathrm{GeV}$ (figure 7).

As a direct application of our results, we derived limits on the decaying gravitino dark matter scenario, both from galaxy clusters observations and from the EGBG. We find that the cluster limits on the gravitino lifetime is $\tau_{3 / 2} \gtrsim 1-2 \times 10^{26} \mathrm{~s}$ for gravitino masses up to $1 \mathrm{TeV}$. The cluster constraint becomes stronger than the gamma-ray line limit at gravitino masses of $m_{3 / 2} \gtrsim 250 \mathrm{GeV}$. As a result, we found limits on the $R$-parity breaking parameter $\zeta$ of the order of $\mathcal{O}\left(10^{-10}\right)$ for gravitino masses $250 \mathrm{GeV} \lesssim m_{3 / 2} \lesssim 1 \mathrm{TeV}$. These constraints were used to set lower limits on NLSP decay lengths corresponding to two different types of universal boundary conditions at the grand unification scale of supergravity models. Interestingly, all the implied decay lengths should be accessible at the LHC.

\section{Acknowledgments}

We thank Hans Böhringer, Julien Lavalle, Luca Maccione, David Paneque, Christopher Savage, Pat Scott, Georg Weiglein and Stephan Zimmer for useful discussions. X.H. was supported by the exchange program between the Max-Planck Society and the Chinese Academy of Science. C.W. thanks the Kavli Institute for Theoretical Physics, Beijing, for warm hospitality during the final stages of this work. This work was partly carried out at the Theory Division of CERN in the context of the TH-Institute DMUH'11 (18-29 July 2011).

\section{References}

[1] F. Zwicky, Die Rotverschiebung von extragalaktischen Nebeln, Helv. Phys. Acta 6 (1933) 110 [INSPIRE].

[2] G. Jungman, M. Kamionkowski and K. Griest, Supersymmetric dark matter, Phys. Rept. 267 (1996) 195 [hep-ph/9506380] [InSPIRE].

[3] L. Bergstrom, Nonbaryonic dark matter: observational evidence and detection methods, Rept. Prog. Phys. 63 (2000) 793 [hep-ph/0002126] [INSPIRE].

[4] G. Bertone, D. Hooper and J. Silk, Particle dark matter: evidence, candidates and constraints, Phys. Rept. 405 (2005) 279 [hep-ph/0404175] [INSPIRE].

[5] R. Essig, N. Sehgal and L.E. Strigari, Bounds on cross-sections and lifetimes for dark matter annihilation and decay into charged leptons from gamma-ray observations of dwarf galaxies, Phys. Rev. D 80 (2009) 023506 [arXiv:0902.4750] [InSPIRE].

[6] P. Scott et al., Direct constraints on minimal supersymmetry from Fermi-LAT observations of the dwarf galaxy Segue 1, JCAP 01 (2010) 031 [arXiv:0909.3300] [INSPIRE].

[7] HESS collaboration, A. Abramowski et al., H.E.S.S. constraints on dark matter annihilations towards the Sculptor and Carina dwarf galaxies, Astropart. Phys. 34 (2011) 608 [arXiv: 1012.5602] [INSPIRE]. 
[8] Fermi LAT collaboration, A.A. Abdo et al., Observations of Milky Way dwarf spheroidal galaxies with the Fermi-LAT detector and constraints on dark matter models, Astrophys. J. 712 (2010) 147 [arXiv:1001.4531] [INSPIRE].

[9] A. Geringer-Sameth and S.M. Koushiappas, Exclusion of canonical WIMPs by the joint analysis of Milky Way dwarfs with Fermi, Phys. Rev. Lett. 107 (2011) 241303 [arXiv: 1108.2914] [INSPIRE].

[10] MAGIC collaboration, J. Aleksic et al., Searches for dark matter annihilation signatures in the Segue 1 satellite galaxy with the MAGIC-I telescope, JCAP 06 (2011) 035 [arXiv: 1103.0477] [INSPIRE].

[11] Fermi-LAT collaboration, M. Ackermann et al., Constraining dark matter models from a combined analysis of Milky Way satellites with the Fermi Large Area Telescope, Phys. Rev. Lett. 107 (2011) 241302 [arXiv:1108.3546] [INSPIRE].

[12] HESS collaboration, F.A. Aharonian et al., H.E.S.S. observations of the galactic center region and their possible dark matter interpretation, Phys. Rev. Lett. 97 (2006) 221102 [Erratum ibid. 97 (2006) 249901] [astro-ph/0610509] [INSPIRE].

[13] G. Dobler, D.P. Finkbeiner, I. Cholis, T.R. Slatyer and N. Weiner, The Fermi haze: a gamma-ray counterpart to the microwave haze, Astrophys. J. 717 (2010) 825 [arXiv: 0910 . 4583] [INSPIRE].

[14] D. Hooper and L. Goodenough, Dark matter annihilation in the galactic center as seen by the Fermi Gamma Ray Space Telescope, Phys. Lett. B 697 (2011) 412 [arXiv:1010.2752] [INSPIRE].

[15] G. Dobler, I. Cholis and N. Weiner, The Fermi gamma-ray haze from dark matter annihilations and anisotropic diffusion, Astrophys. J. 741 (2011) 25 [arXiv:1102.5095] [INSPIRE].

[16] J. Ellis, K.A. Olive and V.C. Spanos, Galactic-centre gamma rays in CMSSM dark matter scenarios, JCAP 10 (2011) 024 [arXiv: 1106.0768] [INSPIRE].

[17] Fermi-LAT collaboration, V. Vitale and A. Morselli, Search for dark matter with Fermi Large Area Telescope: the galactic center, Nucl. Instrum. Meth. A 630 (2011) 147 [INSPIRE].

[18] HESS collaboration, A. Abramowski et al., Search for a dark matter annihilation signal from the galactic center halo with H.E.S.S, Phys. Rev. Lett. 106 (2011) 161301 [arXiv:1103.3266] [INSPIRE].

[19] T. Bringmann, F. Calore, G. Vertongen and C. Weniger, On the relevance of sharp gamma-ray features for indirect dark matter searches, Phys. Rev. D 84 (2011) 103525 [arXiv: 1106.1874] [INSPIRE].

[20] S. Colafrancesco, S. Profumo and P. Ullio, Multi-frequency analysis of neutralino dark matter annihilations in the Coma cluster, Astron. Astrophys. 455 (2006) 21 [astro-ph/0507575] [INSPIRE].

[21] T.E. Jeltema, J. Kehayias and S. Profumo, Gamma rays from clusters and groups of galaxies: cosmic rays versus dark matter, Phys. Rev. D 80 (2009) 023005 [arXiv:0812.0597] [INSPIRE].

[22] E.A. Baltz et al., Pre-launch estimates for GLAST sensitivity to dark matter annihilation signals, JCAP 07 (2008) 013 [arXiv:0806.2911] [INSPIRE].

[23] HESS collaboration, F.A. Aharonian et al., Constraints on the multi-TeV particle population in the Coma galaxy cluster with H.E.S.S. observations, arXiv:0907.0727 [INSPIRE].

[24] MAGIC collaboration, J. Aleksic et al., MAGIC gamma-ray telescope observation of the Perseus cluster of galaxies: implications for cosmic rays, dark matter and NGC1275, Astrophys. J. 710 (2010) 634 [arXiv:0909.3267] [INSPIRE]. 
[25] A. Pinzke, C. Pfrommer and L. Bergstrom, Gamma-rays from dark matter annihilations strongly constrain the substructure in halos, Phys. Rev. Lett. 103 (2009) 181302 [arXiv:0905.1948] [INSPIRE].

[26] FERMi LAT collaboration, M. Ackermann et al., Constraints on dark matter annihilation in clusters of galaxies with the Fermi Large Area Telescope, JCAP 05 (2010) 025 [arXiv: 1002.2239] [INSPIRE].

[27] L. Dugger, T.E. Jeltema and S. Profumo, Constraints on decaying dark matter from Fermi observations of nearby galaxies and clusters, JCAP 12 (2010) 015 [arXiv:1009.5988] [INSPIRE].

[28] Fermi-LAT collaboration, M. Ackermann et al., GeV gamma-ray flux upper limits from clusters of galaxies, arXiv:1006.0748 [INSPIRE].

[29] M.A. Sanchez-Conde, M. Cannoni, F. Zandanel, M.E. Gomez and F. Prada, Dark matter searches with Cherenkov telescopes: nearby dwarf galaxies or local galaxy clusters?, JCAP 12 (2011) 011 [arXiv:1104.3530] [INSPIRE].

[30] A. Pinzke, C. Pfrommer and L. Bergstrom, Prospects of detecting gamma-ray emission from galaxy clusters: cosmic rays and dark matter annihilations, Phys. Rev. D 84 (2011) 123509 [arXiv:1105.3240] [INSPIRE].

[31] W. Buchmüller, L. Covi, K. Hamaguchi, A. Ibarra and T. Yanagida, Gravitino dark matter in R-parity breaking vacua, JHEP 03 (2007) 037 [hep-ph/0702184] [INSPIRE].

[32] F. Takayama and M. Yamaguchi, Gravitino dark matter without R-parity, Phys. Lett. B 485 (2000) 388 [hep-ph/0005214] [INSPIRE].

[33] G. Bertone, W. Buchmüller, L. Covi and A. Ibarra, Gamma-rays from decaying dark matter, JCAP 11 (2007) 003 [arXiv:0709.2299] [INSPIRE].

[34] A. Ibarra and D. Tran, Gamma ray spectrum from gravitino dark matter decay, Phys. Rev. Lett. 100 (2008) 061301 [arXiv:0709.4593] [INSPIRE].

[35] S. Lola, P. Osland and A.R. Raklev, Radiative gravitino decays from R-parity violation, Phys. Lett. B 656 (2007) 83 [arXiv:0707.2510] [INSPIRE].

[36] A. Ibarra and D. Tran, Antimatter signatures of gravitino dark matter decay, JCAP 07 (2008) 002 [arXiv:0804.4596] [INSPIRE].

[37] K. Ishiwata, S. Matsumoto and T. Moroi, High energy cosmic rays from the decay of gravitino dark matter, Phys. Rev. D 78 (2008) 063505 [arXiv:0805.1133] [INSPIRE].

[38] K. Ishiwata, T. Ito and T. Moroi, Long-lived unstable superparticles at the LHC, Phys. Lett. B 669 (2008) 28 [arXiv:0807.0975] [InSPIRE].

[39] L. Covi, M. Grefe, A. Ibarra and D. Tran, Unstable gravitino dark matter and neutrino flux, JCAP 01 (2009) 029 [arXiv: 0809.5030] [InSPIRE].

[40] W. Buchmüller, A. Ibarra, T. Shindou, F. Takayama and D. Tran, Probing gravitino dark matter with PAMELA and Fermi, JCAP 09 (2009) 021 [arXiv:0906.1187] [INSPIRE].

[41] K.-Y. Choi, D.E. Lopez-Fogliani, C. Muñoz and R.R. de Austri, Gamma-ray detection from gravitino dark matter decay in the $\mu \nu S S M, J C A P 03$ (2010) 028 [arXiv:0906.3681] [INSPIRE].

[42] N.-E. Bomark, S. Lola, P. Osland and A. Raklev, Photon, neutrino and charged particle spectra from R-violating gravitino decays, Phys. Lett. B 686 (2010) 152 [arXiv:0911.3376] [INSPIRE].

[43] K.-Y. Choi and C.E. Yaguna, New decay modes of gravitino dark matter, Phys. Rev. D 82 (2010) 015008 [arXiv: 1003.3401] [inSPIRE].

[44] K.-Y. Choi, D. Restrepo, C.E. Yaguna and O. Zapata, Indirect detection of gravitino dark matter including its three-body decays, JCAP 10 (2010) 033 [arXiv:1007.1728] [INSPIRE]. 
[45] Fermi LAT collaboration, W.B. Atwood et al., The Large Area Telescope on the Fermi Gamma-ray Space Telescope mission, Astrophys. J. 697 (2009) 1071 [arXiv:0902.1089] [INSPIRE].

[46] Fermi LAT collaboration, A.A. Abdo et al., Fermi LAT search for photon lines from 30 to 200 GeV and dark matter implications, Phys. Rev. Lett. 104 (2010) 091302 [arXiv: 1001.4836] [INSPIRE].

[47] G. Vertongen and C. Weniger, Hunting dark matter gamma-ray lines with the Fermi LAT, JCAP 05 (2011) 027 [arXiv:1101.2610] [INSPIRE].

[48] D. Eichler, TeV particles as weakly unstable dark matter, Phys. Rev. Lett. 63 (1989) 2440 [INSPIRE].

[49] A. Arvanitaki et al., Astrophysical probes of unification, Phys. Rev. D 79 (2009) 105022 [arXiv:0812.2075] [INSPIRE].

[50] C. Arina, T. Hambye, A. Ibarra and C. Weniger, Intense gamma-ray lines from hidden vector dark matter decay, JCAP 03 (2010) 024 [arXiv:0912.4496] [INSPIRE].

[51] PAMELA collaboration, O. Adriani et al., An anomalous positron abundance in cosmic rays with energies 1.5-100 GeV, Nature 458 (2009) 607 [arXiv: 0810.4995] [INSPIRE].

[52] Fermi LAT collaboration, A.A. Abdo et al., Measurement of the cosmic ray $e^{+}+e^{-}$ spectrum from $20 \mathrm{GeV}$ to $1 \mathrm{TeV}$ with the Fermi Large Area Telescope, Phys. Rev. Lett. 102 (2009) 181101 [arXiv:0905.0025] [INSPIRE].

[53] Fermi LAT collaboration, M. Ackermann et al., Fermi LAT observations of cosmic-ray electrons from 7 GeV to 1 TeV, Phys. Rev. D 82 (2010) 092004 [arXiv:1008.3999] [InSPIRE].

[54] HESS collaboration, F.A. Aharonian et al., The energy spectrum of cosmic-ray electrons at TeV energies, Phys. Rev. Lett. 101 (2008) 261104 [arXiv:0811.3894] [INSPIRE].

[55] HESS collaboration, F.A. Aharonian et al., Probing the ATIC peak in the cosmic-ray electron spectrum with H.E.S.S, Astron. Astrophys. 508 (2009) 561 [arXiv:0905.0105] [INSPIRE].

[56] PAMELA collaboration, O. Adriani et al., PAMELA results on the cosmic-ray antiproton flux from $60 \mathrm{MeV}$ to $180 \mathrm{GeV}$ in kinetic energy, Phys. Rev. Lett. 105 (2010) 121101 [arXiv: 1007.0821] [INSPIRE].

[57] P.J. Fox and E. Poppitz, Leptophilic dark matter, Phys. Rev. D 79 (2009) 083528 [arXiv: 0811.0399] [INSPIRE].

[58] B. Kyae, PAMELA/ATIC anomaly from the meta-stable extra dark matter component and the leptophilic Yukawa interaction, JCAP 07 (2009) 028 [arXiv:0902.0071] [INSPIRE].

[59] A. Ibarra, A. Ringwald, D. Tran and C. Weniger, Cosmic rays from leptophilic dark matter decay via kinetic mixing, JCAP 08 (2009) 017 [arXiv:0903.3625] [INSPIRE].

[60] X.-J. Bi, X.-G. He and Q. Yuan, Parameters in a class of leptophilic models from PAMELA, ATIC and FERMI, Phys. Lett. B 678 (2009) 168 [arXiv:0903.0122] [InSPIRE].

[61] D. Spolyar, M.R. Buckley, K. Freese, D. Hooper and H. Murayama, High energy neutrinos as a test of leptophilic dark matter, arXiv:0905.4764 [INSPIRE].

[62] T. Cohen and K.M. Zurek, Leptophilic dark matter from the lepton asymmetry, Phys. Rev. Lett. 104 (2010) 101301 [arXiv:0909.2035] [INSPIRE].

[63] E.J. Chun, J.-C. Park and S. Scopel, Dirac gaugino as leptophilic dark matter, JCAP 02 (2010) 015 [arXiv:0911.5273] [INSPIRE].

[64] A. Ibarra, D. Tran and C. Weniger, Decaying dark matter in light of the PAMELA and Fermi LAT data, JCAP 01 (2010) 009 [arXiv:0906.1571] [INSPIRE].

[65] P. Meade, M. Papucci, A. Strumia and T. Volansky, Dark matter interpretations of the $e^{ \pm}$ excesses after FERMI, Nucl. Phys. B 831 (2010) 178 [arXiv:0905.0480] [InSPIRE]. 
[66] Q. Yuan, P.-F. Yin, X.-J. Bi, X.-M. Zhang and S.-H. Zhu, Gamma rays and neutrinos from dark matter annihilation in galaxy clusters, Phys. Rev. D 82 (2010) 023506 [arXiv: 1002.0197] [INSPIRE].

[67] J. Ke, M. Luo, L. Wang and G. Zhu, Gamma-rays from nearby clusters: constraints on selected decaying dark matter models, Phys. Lett. B 698 (2011) 44 [arXiv:1101.5878] [INSPIRE].

[68] M. Luo, L. Wang and G. Zhu, A model independent method to study dark matter induced leptons and gamma rays, arXiv:1107.3024 [INSPIRE].

[69] T.H. Reiprich and H. Boehringer, The mass function of an X-ray flux-limited sample of galaxy clusters, Astrophys. J. 567 (2002) 716 [astro-ph/0111285] [INSPIRE].

[70] Y. Chen, T.H. Reiprich, H. Böhringer, Y. Ikebe and Y.-Y. Zhang, Statistics of X-ray observables for the cooling-core and non-cooling core galaxy clusters, Astron. Astrophys. 466 (2007) 805 [astro-ph/0702482] [INSPIRE].

[71] W. Voges et al., The ROSAT all-sky survey bright source catalogue, Astron. Astrophys. 349 (1999) 389 [astro-ph/9909315] [INSPIRE].

[72] WMAP collaboration, E. Komatsu et al., Seven-year Wilkinson Microwave Anisotropy Probe (WMAP) observations: cosmological interpretation, Astrophys. J. Suppl. 192 (2011) 18 [arXiv: 1001.4538] [INSPIRE].

[73] Fermi LAT collaboration, A.A. Abdo et al., Fermi Large Area Telescope gamma-ray detection of the radio galaxy M87, Astrophys. J. 707 (2009) 55 [arXiv:0910.3565] [INSPIRE].

[74] LAT collaboration, A.A. Abdo et al., Fermi discovery of gamma-ray emission from NGC1275, Astrophys. J. 699 (2009) 31 [arXiv:0904.1904] [INSPIRE].

[75] T. Sjöstrand, S. Mrenna and P.Z. Skands, PYTHIA 6.4 physics and manual, JHEP 05 (2006) 026 [hep-ph/0603175] [INSPIRE].

[76] J.A.R. Cembranos, A. de la Cruz-Dombriz, A. Dobado, R.A. Lineros and A.L. Maroto, Photon spectra from WIMP annihilation, Phys. Rev. D 83 (2011) 083507 [arXiv:1009.4936] [INSPIRE].

[77] G.R. Blumenthal and R.J. Gould, Bremsstrahlung, synchrotron radiation and Compton scattering of high-energy electrons traversing dilute gases, Rev. Mod. Phys. 42 (1970) 237 [INSPIRE].

[78] T.A. Ensslin, C. Pfrommer, F. Miniati and K. Subramanian, Cosmic ray transport in galaxy clusters: implications for radio halos, gamma-ray signatures and cool core heating, arXiv: 1008.4717 [INSPIRE].

[79] P. Blasi and S. Colafrancesco, Cosmic rays, radio halos and non-thermal X-ray emission in clusters of galaxies, Astropart. Phys. 12 (1999) 169 [astro-ph/9905122] [INSPIRE].

[80] J. Lavalle, C. Boehm and J. Barthes, On the Sunyaev-Zel'dovich effect from dark matter annihilation or decay in galaxy clusters, JCAP 02 (2010) 005 [arXiv:0907.5589] [INSPIRE].

[81] http://www.slac.stanford.edu/exp/glast/groups/canda/lat_Performance.htm.

[82] J.F. Navarro, C.S. Frenk and S.D.M. White, A universal density profile from hierarchical clustering, Astrophys. J. 490 (1997) 493 [astro-ph/9611107] [INSPIRE].

[83] A.W. Graham, D. Merritt, B. Moore, J. Diemand and B. Terzic, Empirical models for dark matter halos. I. Nonparametric construction of density profiles and comparison with parametric models, Astron. J. 132 (2006) 2685 [astro-ph/0509417] [INSPIRE].

[84] J.F. Navarro et al., The diversity and similarity of cold dark matter halos, arXiv:0810.1522 [INSPIRE].

[85] D.A. Buote et al., The X-ray concentration-virial mass relation, Astrophys. J. 664 (2007) 123 [astro-ph/0610135] [INSPIRE]. 
[86] W. Hu and A.V. Kravtsov, Sample variance considerations for cluster surveys, Astrophys. J. 584 (2003) 702 [astro-ph/0203169] [INSPIRE].

[87] A.R. Duffy, J. Schaye, S.T. Kay and C. Dalla Vecchia, Dark matter halo concentrations in the Wilkinson Microwave Anisotropy Probe year 5 cosmology, Mon. Not. Roy. Astron. Soc. 390 (2008) L64 [arXiv:0804.2486] [INSPIRE].

[88] A. Klypin, S. Trujillo-Gomez and J. Primack, Halos and galaxies in the standard cosmological model: results from the Bolshoi simulation, arXiv:1002.3660 [INSPIRE].

[89] S. Hofmann, D.J. Schwarz and H. Stoecker, Damping scales of neutralino cold dark matter, Phys. Rev. D 64 (2001) 083507 [astro-ph/0104173] [InSPIRE].

[90] A.M. Green, S. Hofmann and D.J. Schwarz, The first WIMPy halos, JCAP 08 (2005) 003 [astro-ph/0503387] [INSPIRE].

[91] T. Bringmann, Particle models and the small-scale structure of dark matter, New J. Phys. 11 (2009) 105027 [arXiv: 0903.0189] [InSPIRE].

[92] V. Springel et al., The Aquarius Project: the subhalos of galactic halos, Mon. Not. Roy. Astron. Soc. 391 (2008) 1685 [arXiv:0809.0898] [INSPIRE].

[93] V. Springel et al., A blueprint for detecting supersymmetric dark matter in the galactic halo, arXiv:0809.0894 [INSPIRE].

[94] M. Kuhlen, J. Diemand, P. Madau and M. Zemp, The Via Lactea INCITE simulation: galactic dark matter substructure at high resolution, J. Phys. Conf. Ser. 125 (2008) 012008 [arXiv:0810.3614] [INSPIRE].

[95] E. Contini, G. De Lucia and S. Borgani, Statistics of substructures in dark matter haloes, arXiv:1111.1911 [INSPIRE].

[96] L. Gao, C.S. Frenk, A. Jenkins, V. Springel and S.D.M. White, Where will supersymmetric dark matter first be seen?, Mon. Not. Roy. Astron. Soc. 419 (2012) 1721 [arXiv:1107.1916] [INSPIRE].

[97] M. Su, T.R. Slatyer and D.P. Finkbeiner, Giant gamma-ray bubbles from Fermi-LAT: AGN activity or bipolar galactic wind?, Astrophys. J. $\mathbf{7 2 4}$ (2010) 1044 [arXiv:1005.5480] [INSPIRE].

[98] Fermi LAT collaboration, A.A. Abdo et al., Fermi Large Area Telescope second source catalog, arXiv: 1108.1435 [INSPIRE].

[99] W.A. Rolke, A.M. Lopez and J. Conrad, Limits and confidence intervals in the presence of nuisance parameters, Nucl. Instrum. Meth. A 551 (2005) 493 [physics/0403059] [INSPIRE].

[100] J. Conrad, T. Ylinen and J. Scargle, Statistical analysis of detection of, and upper limits on, dark matter lines, AIP Conf. Proc. 921 (2007) 586 [InSPIRE].

[101] Fermi-LAT collaboration, A.A. Abdo et al., The spectrum of the isotropic diffuse gamma-ray emission derived from first-year Fermi Large Area Telescope data, Phys. Rev. Lett. 104 (2010) 101101 [arXiv:1002.3603] [INSPIRE].

[102] S. Matsumoto, K. Ishiwata and T. Moroi, Cosmic gamma-ray from inverse Compton process in unstable dark matter scenario, Phys. Lett. B 679 (2009) 1 [arXiv:0905.4593] [INSPIRE].

[103] M. Papucci and A. Strumia, Robust implications on dark matter from the first FERMI sky gamma map, JCAP 03 (2010) 014 [arXiv:0912.0742] [INSPIRE].

[104] M. Cirelli, P. Panci and P.D. Serpico, Diffuse gamma ray constraints on annihilating or decaying dark matter after Fermi, Nucl. Phys. B 840 (2010) 284 [arXiv:0912.0663] [INSPIRE].

[105] M. Pohl and D. Eichler, Fermi constrains dark matter origin of high energy positron anomaly, arXiv:0912.1203 [INSPIRE]. 
[106] L. Zhang, C. Weniger, L. Maccione, J. Redondo and G. Sigl, Constraining decaying dark matter with Fermi LAT gamma-rays, JCAP 06 (2010) 027 [arXiv:0912.4504] [INSPIRE].

[107] F.W. Stecker, M.A. Malkan and S.T. Scully, Intergalactic photon spectra from the far IR to the UV Lyman limit for $0<Z<6$ and the optical depth of the universe to high energy gamma-rays, Astrophys. J. 648 (2006) 774 [astro-ph/0510449] [INSPIRE].

[108] The GBM collaboration, A.A. Abdo et al., Fermi Large Area Telescope constraints on the gamma-ray opacity of the universe, Astrophys. J. 723 (2010) 1082 [arXiv:1005.0996] [INSPIRE].

[109] A. Ibarra, D. Tran and C. Weniger, Detecting gamma-ray anisotropies from decaying dark matter: prospects for Fermi LAT, Phys. Rev. D 81 (2010) 023529 [arXiv:0909.3514] [INSPIRE].

[110] Fermi-LAT collaboration, A.A. Abdo et al., Constraints on cosmological dark matter annihilation from the Fermi-LAT isotropic diffuse gamma-ray measurement, JCAP 04 (2010) 014 [arXiv: 1002.4415] [INSPIRE].

[111] K.N. Abazajian, S. Blanchet and J.P. Harding, Current and future constraints on dark matter from prompt and inverse-Compton photon emission in the isotropic diffuse gamma-ray background, arXiv:1011.5090 [INSPIRE].

[112] F. Calore, V. De Romeri and F. Donato, Conservative upper limits on WIMP annihilation cross section from Fermi-LAT gamma-rays, arXiv:1105.4230 [INSPIRE].

[113] Fermi LAT collaboration, M. Ackermann et al., Measurement of separate cosmic-ray electron and positron spectra with the Fermi Large Area Telescope, arXiv:1109.0521 [INSPIRE].

[114] D.E. McLaughlin, Evidence in Virgo for the universal dark matter halo, astro-ph/9812242 [INSPIRE].

[115] S. Schindler, B. Binggeli and H. Boehringer, Morphology of the Virgo cluster: gas versus galaxies, astro-ph/9811464 [INSPIRE].

[116] M. Paolillo, G. Fabbiano, G. Peres and D.-W. Kim, Deep ROSAT-HRI observations of the NGC1399/NGC1404 region: morphology and structure of the X-ray halo, Astrophys. J. 565 (2002) 883 [astro-ph/0109342] [INSPIRE].

[117] J.D. Murphy, K. Gebhardt and J.J. Adams, Galaxy kinematics with VIRUS-P: the dark matter halo of M87, Astrophys. J. 729 (2011) 129 [arXiv:1101.1957] [INSPIRE].

[118] M.J. Drinkwater, M.D. Gregg and M. Colless, Substructure and dynamics of the Fornax cluster, astro-ph/0012415 [INSPIRE].

[119] P. Cote, D.E. McLaughlin, J.G. Cohen and J.P. Blakeslee, Dynamics of the globular cluster system associated with M49 (NGC4472): cluster orbital properties and the distribution of dark matter, Astrophys. J. 591 (2003) 850 [astro-ph/0303229] [INSPIRE].

[120] N. Okabe, Y. Okura and T. Futamase, Weak lensing mass measurements of substructures in COMA cluster with Subaru/Suprime-Cam, Astrophys. J. 713 (2010) 291 [arXiv:1001.2402] [INSPIRE].

[121] A. Bonafede et al., The Coma cluster magnetic field from Faraday rotation measures, arXiv: 1002.0594 [INSPIRE].

[122] A. Bonafede et al., Fractional polarization and magnetic fields in the ICM, arXiv:1103.0277 [INSPIRE].

[123] H. Pagels and J.R. Primack, Supersymmetry, cosmology and new TeV physics, Phys. Rev. Lett. 48 (1982) 223 [INSPIRE].

[124] M. Bolz, A. Brandenburg and W. Buchmüller, Thermal production of gravitinos, Nucl. Phys. B 606 (2001) 518 [Erratum ibid. B 790 (2008) 336] [hep-ph/0012052] [INSPIRE]. 
[125] J. Pradler and F.D. Steffen, Thermal gravitino production and collider tests of leptogenesis, Phys. Rev. D 75 (2007) 023509 [hep-ph/0608344] [INSPIRE].

[126] W. Buchmüller, M. Endo and T. Shindou, Superparticle mass window from leptogenesis and decaying gravitino dark matter, JHEP 11 (2008) 079 [arXiv:0809.4667] [INSPIRE].

[127] J. Pradler and F.D. Steffen, Constraints on the reheating temperature in gravitino dark matter scenarios, Phys. Lett. B 648 (2007) 224 [hep-ph/0612291] [INSPIRE].

[128] K. Nakayama, F. Takahashi and T.T. Yanagida, Constraint on the gravitino mass in hybrid inflation, JCAP 12 (2010) 010 [arXiv: 1007.5152] [INSPIRE].

[129] M. Fukugita and T. Yanagida, Baryogenesis without grand unification, Phys. Lett. B 174 (1986) 45 [INSPIRE].

[130] W. Buchmüller, P. Di Bari and M. Plümacher, Leptogenesis for pedestrians, Annals Phys. 315 (2005) 305 [hep-ph/0401240] [INSPIRE].

[131] J.R. Ellis, D.V. Nanopoulos and S. Sarkar, The cosmology of decaying gravitinos, Nucl. Phys. B 259 (1985) 175 [InSPIRE].

[132] S. Sarkar, Big bang nucleosynthesis and physics beyond the standard model, Rept. Prog. Phys. 59 (1996) 1493 [hep-ph/9602260] [INSPIRE].

[133] M. Kawasaki, K. Kohri and T. Moroi, Big-bang nucleosynthesis and hadronic decay of long-lived massive particles, Phys. Rev. D 71 (2005) 083502 [astro-ph/0408426] [INSPIRE].

[134] M. Pospelov, Particle physics catalysis of thermal big bang nucleosynthesis, Phys. Rev. Lett. 98 (2007) 231301 [hep-ph/0605215] [INSPIRE].

[135] K. Hamaguchi, T. Hatsuda, M. Kamimura, Y. Kino and T.T. Yanagida, Stau-catalyzed ${ }^{6}$ Li production in big-bang nucleosynthesis, Phys. Lett. B 650 (2007) 268 [hep-ph/0702274] [INSPIRE].

[136] M. Pospelov, J. Pradler and F.D. Steffen, Constraints on supersymmetric models from catalytic primordial nucleosynthesis of beryllium, JCAP 11 (2008) 020 [arXiv:0807.4287] [INSPIRE].

[137] M. Kawasaki, K. Kohri, T. Moroi and A. Yotsuyanagi, Big-bang nucleosynthesis and gravitino, Phys. Rev. D 78 (2008) 065011 [arXiv:0804.3745] [INSPIRE].

[138] S. Bobrovskyi, W. Buchmüller, J. Hajer and J. Schmidt, Broken R-parity in the sky and at the LHC, JHEP 10 (2010) 061 [arXiv: 1007.5007] [INSPIRE].

[139] Particle Data Group collaboration, K. Nakamura et al., Review of particle physics, J. Phys. G 37 (2010) 075021 [INSPIRE].

[140] ATLAS collaboration, G. Aad et al., Combined exclusion reach of searches for squarks and gluinos using final states with jets, missing transverse momentum, and zero or one lepton, with the ATLAS detector in $\sqrt{s}=7$ TeV proton-proton collisions, ATLAS-CONF-2011-064 (2011).

[141] M.A. Diaz, S.G. Saenz and B. Koch, Gravitino dark matter and neutrino masses in partial split supersymmetry, Phys. Rev. D 84 (2011) 055007 [arXiv:1106.0308] [INSPIRE].

[142] S. Bobrovskyi, W. Buchmüller, J. Hajer and J. Schmidt, Quasi-stable neutralinos at the LHC, JHEP 09 (2011) 119 [arXiv: 1107.0926] [INSPIRE].

[143] B. Mukhopadhyaya, S. Roy and F. Vissani, Correlation between neutrino oscillations and collider signals of supersymmetry in an R-parity violating model, Phys. Lett. B 443 (1998) 191 [hep-ph/9808265] [INSPIRE].

[144] E.J. Chun and J.S. Lee, Implication of super-Kamiokande data on R-parity violation, Phys. Rev. D 60 (1999) 075006 [hep-ph/9811201] [INSPIRE]. 